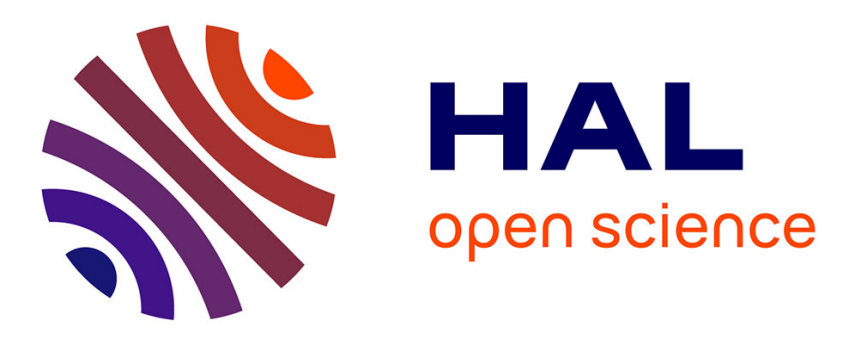

\title{
Stability and Convergence Analysis of Time-domain Perfectly Matched Layers for The Wave Equation in Waveguides
}

\author{
Eliane Bécache, Maryna Kachanovska
}

\section{To cite this version:}

Eliane Bécache, Maryna Kachanovska. Stability and Convergence Analysis of Time-domain Perfectly Matched Layers for The Wave Equation in Waveguides. SIAM Journal on Numerical Analysis, 2021, 10.1137/20M1330543 . hal-02536375

\section{HAL Id: hal-02536375 \\ https://hal.science/hal-02536375}

Submitted on 8 Apr 2020

HAL is a multi-disciplinary open access archive for the deposit and dissemination of scientific research documents, whether they are published or not. The documents may come from teaching and research institutions in France or abroad, or from public or private research centers.
L'archive ouverte pluridisciplinaire HAL, est destinée au dépôt et à la diffusion de documents scientifiques de niveau recherche, publiés ou non, émanant des établissements d'enseignement et de recherche français ou étrangers, des laboratoires publics ou privés. 


\title{
STABILITY AND CONVERGENCE ANALYSIS OF TIME-DOMAIN PERFECTLY MATCHED LAYERS FOR THE WAVE EQUATION IN WAVEGUIDES
}

\author{
ELIANE BÉCACHE * AND MARYNA KACHANOVSKA *
}

\begin{abstract}
This work is dedicated to the proof of stability and convergence of the Bérenger's perfectly matched layers in the waveguides for an arbitrary $L^{\infty}$ damping function. The proof relies on the Laplace domain techniques and an explicit representation of the solution to the PML problem in the waveguide. A bound for the PML error that depends on the absorption parameter and the length of the PML is presented. Numerical experiments confirm the theoretical findings.
\end{abstract}

Key words. wave equation, perfectly matched layers, waveguide, Laplace transform, Dirichletto-Neumann operator

AMS subject classifications. 65M12, 35L05

1 Introduction The perfectly matched layer method (PMLs) was introduced by J.-P. Berenger for simulating transient wave propagation in unbounded domains described by 2D Maxwell's equations [17](in 1994) and 3D Maxwell's equations in [18](in 1996). Since then it had gained popularity in the engineering and physics communities, because of its efficiency and ease of implementation, see e.g. [35, 57, 49].

Compared to other existing methods of handling the unboundedness of the computational domain, the PML method of course has its advantages and disadvantages. For example, unlike when using absorbing boundary conditions [34, 43, 26, 39, 40, 38, 42], the application of the PMLs does not require any special handling of the corners $[39,8,53]$. Let us remark that this issue had been overcome, at least partially, by double absorbing boundary conditions $[37,6]$.

For many problems the PMLs remain more computationally efficient than the boundary integral operators for computing transparent boundary conditions [3, 9]. Formulating the PML system suitable for computational purposes does not require any auxiliary knowledge (e.g. a computable form of the fundamental solution) but the underlying PDEs in the explicit form. Unlike the pole condition-based methods, see e.g. [44] and references therein, and half-space matching methods, cf. [19], which are still at early stages of their development and predominantly have been applied in the frequency regime, the PMLs have been successfully used for transient problems.

However, PMLs are known to produce instabilities when applied to anisotropic $[12,45,31,54,2]$ or dispersive $[15,16,14]$ media. Some of those have been overcome in the above-mentioned works, however, the question of stabilizing the PMLs remains model-dependent. Moreover, even in situations when the PMLs remain stable, their error control is rather difficult, because of the interplay of the various parameters of the PML and the discretization errors, see [28, 52, 5, 25].

Finally, from the point of view of the mathematical analysis of the PMLs, there are still some gaps remaining. Much progress had been done in in-depth studies of the PMLs in the frequency domain: for example, the questions of the well-posedness and error analysis of the PMLs were treated in [22, 21, 24, 51, 10, 11]; the numerical analysis was performed in particular in $[23,20,50]$. While there had been a lot of advancements in the analysis of the time-domain PMLs, see e.g. [13, 12, 4, 1],

*POEMS (UMR 7231 CNRS-ENSTA-INRIA), INRIA Saclay, Institut Polytechnique de Paris, Palaiseau, France (eliane.becache@inria.fr, maryna.kachanovska@inria.fr). 
for many problems, the stability for non-constant absorption parameters and the convergence analysis of the time-domain PMLs remains an open question. Often $[13,12,33]$ the stability analysis is done in a simplified setting when all the absorption parameters are constant. The case of variable absorption parameter had been treated in e.g. [13, 46, 41], however, the estimates in these works do not imply stability of the PML system. Up to our knowledge, the only work where the stability for arbitrary absorption parameter and the convergence of the Cartesian PMLs in the time domain had been proven is the article by J. Diaz and P. Joly [32]. There, the authors construct an explicit fundamental solution for the PML system for the 2D acoustic wave equation, based on the Cagniard-de-Hoop contour deformation method and, crucially, on the method of reflections. Then they derive convergence estimates for the PMLs, which, according to the numerical experiments, are close to optimal.

The subject of the present work is the stability and convergence analysis of the time-domain PMLs for the wave equation in 3D waveguides, where it is not possible to use the above mentioned techniques for computing the Green function in the explicit form, since, in particular, the method of reflections can no longer be applied. Our well-posedness/stability analysis will be based on the modal decompositions and some energy-like Laplace domain arguments, while the finer stability and convergence analysis will exploit an exact representation of the solution in a 3D waveguide.

The article is divided into the following main parts:

- in Section 2 we present the problem, introduce notations, recall the PML method;

- Section 3 is dedicated to the well-posedness and stability analysis of the PMLs;

- in Section 4 we prove convergence estimates for the PMLs in the time domain;

- Section 5 contains numerical studies of optimality of the estimates of Section 4;

- in Section 6 we outline the results of the article and discuss possible extensions of the techniques used in the paper.

\section{Problem setting and the method of Perfectly Matched Layers.}

\subsection{The wave equation in a $3 \mathrm{D}$ waveguide.}

2.1.1 The problem setting. We look for a solution $u$ of the wave equation in an infinite waveguide $\Omega_{\infty}:=\mathbb{R} \times \mathcal{S}$ (with $\mathcal{S}$ being a Lipschitz bounded domain in $\mathbb{R}^{d}$, $d=1,2)$. We are interested in finding the restriction of the solution $u$ to $\Omega$, which is a bounded domain $\Omega=I \times \mathcal{S}$, where $I=(-a, a)$, for $a>0$. To formulate the problem, let us start with an assumption on the support and regularity of the data.

Assumption 1. The data $f: \mathbb{R}_{+} \times \Omega_{\infty} \rightarrow \mathbb{R}, u_{0}, u_{1}: \Omega_{\infty} \rightarrow \mathbb{R}$ satisfy:

$$
\begin{aligned}
& \text { for all } t \geq 0, \quad \operatorname{supp} f(t) \subset \Omega ; \quad \operatorname{supp} u_{0}, \operatorname{supp} u_{1} \subset \Omega ; \\
& u_{0} \in H^{1}(\Omega), \quad u_{1} \in L^{2}(\Omega), \quad f \in L^{1}\left(0, \infty ; L^{2}(\Omega)\right) .
\end{aligned}
$$

Given $u_{0}, u_{1}, f$ satisfying Assumption 1 , we look for $u: \mathbb{R}_{+} \times \Omega_{\infty} \rightarrow \mathbb{R}$ that satisfies (with $\partial_{\nu}=\boldsymbol{\nu} \cdot \nabla$ and $\boldsymbol{\nu}$ being the exterior normal to $\Omega_{\infty}$ ):

$$
\begin{aligned}
& \partial_{t}^{2} u(t, \mathbf{x})-\Delta u(t, \mathbf{x})=f(t, \mathbf{x}), \quad \mathbf{x} \in \Omega_{\infty}, \\
& \partial_{\nu} u=0 \text { on } \mathbb{R} \times \partial \mathcal{S}, \\
& \left.u\right|_{t=0}=u_{0},\left.\quad \partial_{t} u\right|_{t=0}=u_{1} \text { in } \Omega_{\infty} .
\end{aligned}
$$

The problem (2.2) is well-posed and stable, cf. [30, 55]. In the sequel the expression $a \lesssim b$ will be used in place of $a \leq C b$, for a constant $C>0$ independent of the problem parameters. 
Theorem 2.1 (Well-posedness and stability of (2.2)). Provided $u_{0}, u_{1}, f$ satisfying Assumption 1, for all $T>0$, there exists a unique solution u to (2.2)

$$
u \in C^{1}\left([0, T] ; L^{2}\left(\Omega_{\infty}\right)\right) \cap C^{0}\left([0, T] ; H^{1}\left(\Omega_{\infty}\right)\right) .
$$

This solution satisfies

$$
\begin{aligned}
& \left\|\partial_{t} u\right\|_{L^{2}\left(0, T ; L^{2}(\Omega)\right)}+\|\nabla u\|_{L^{2}\left(0, T ; L^{2}(\Omega)\right)} \lesssim T^{\frac{1}{2}} E_{d}(T), \\
& E_{d}(T):=\left\|\nabla u_{0}\right\|_{L^{2}(\Omega)}+\left\|u_{1}\right\|_{L^{2}(\Omega)}+\|f\|_{L^{1}\left(0, T ; L^{2}(\Omega)\right)} .
\end{aligned}
$$

In the above the index $d$ in $E_{d}$ stands for 'data'.

2.1.2 Sobolev spaces in waveguides. Later we will make use of the decomposition of functions $v: \Omega_{\infty} \rightarrow \mathbb{C}$ in the eigenfunctions of the transverse Laplacian:

$$
\begin{aligned}
& \Delta_{\perp} v=\partial_{y}^{2} v+\partial_{z}^{2} v, \quad \Delta_{\perp}: \mathcal{D}\left(\Delta_{\perp}\right) \rightarrow L^{2}(\mathcal{S}), \quad \text { where } \\
& \mathcal{D}\left(\Delta_{\perp}\right)=\left\{v \in H_{\Delta_{\perp}}^{1}(\mathcal{S}), \quad \partial_{\nu} v=0\right\}, \quad H_{\Delta_{\perp}}^{1}=\left\{v \in H^{1}(\mathcal{S}): \Delta_{\perp} v \in L^{2}(\mathcal{S})\right\} .
\end{aligned}
$$

Because the resolvent of $\Delta_{\perp}$ is compact, its spectrum is discrete, of finite multiplicity, and has infinity as an accumulation point:

$$
-\Delta_{\perp} \phi_{n}=\lambda_{n}^{2} \phi_{n}, \quad 0 \leq \lambda_{0}^{2} \leq \lambda_{1}^{2} \leq \ldots
$$

The eigenfunctions are normalized so that $\left\|\phi_{n}\right\|_{L^{2}(\mathcal{S})}=1$. Moreover,

$$
\int_{\mathcal{S}} \phi_{n} \phi_{m}=\delta_{n, m}, \text { and } \int_{\mathcal{S}} \nabla_{\perp} \phi_{n} \cdot \nabla_{\perp} \phi_{m}=\lambda_{n}^{2} \delta_{n, m},
$$

where $\nabla_{\perp} v=\left(\partial_{y} v, \partial_{z} v\right)^{t}$. By the spectral theorem for self-adjoint operators [29, Chapters VIII.3, VIII.4], any $v \in L^{2}\left(\Omega_{\infty}\right)$ can be decomposed into the Fourier series

$$
\text { A. e. } x \in \mathbb{R}, \quad v(x, .)=\sum_{n=0}^{\infty} v_{n}(x) \phi_{n}, \quad \text { in } L^{2}(\mathcal{S}) .
$$

The above series converges in $D\left(\Delta_{\perp}\right)$, a.e. $x \in \mathbb{R}$; for functions in $L^{2}\left(\Omega_{\infty}\right)$ and $H^{1}\left(\Omega_{\infty}\right)$ the convergence holds in respectively $L^{2}\left(\Omega_{\infty}\right)$ and $H^{1}\left(\Omega_{\infty}\right)$ norms.

Given $\mathcal{O}=I_{\alpha} \times \mathcal{S}, I_{\alpha}=(-\alpha, \alpha), \alpha>0$, the Sobolev norms on $\mathcal{O}$ are:

$$
\|v\|_{L^{2}(\mathcal{O})}^{2}=\sum_{n=0}^{\infty}\left\|v_{n}\right\|_{L^{2}\left(I_{\alpha}\right)}^{2}, \quad\|v\|_{H^{1}(\mathcal{O})}^{2}=\sum_{n=0}^{\infty}\left(1+\lambda_{n}^{2}\right)\left\|v_{n}\right\|_{L^{2}\left(I_{\alpha}\right)}^{2}+\sum_{n=0}^{\infty}\left\|\partial_{x} v_{n}\right\|_{L^{2}\left(I_{\alpha}\right)}^{2} .
$$

The antidual space of $H^{1}(\mathcal{O})$, namely $\widetilde{H}^{-1}(\mathcal{O})$, can be characterized with the help of the Riesz theorem, by associating to each $\widetilde{F} \in \widetilde{H}^{-1}(\mathcal{O})$ a function $F \in H^{1}(\mathcal{O})$ :

$$
\begin{aligned}
\langle\widetilde{F}, v\rangle_{\widetilde{H}^{-1}(\mathcal{O}), H^{1}(\mathcal{O})} & =(F, v)_{H^{1}(\mathcal{O})} \\
& \equiv \sum_{m=0}^{\infty}\left(1+\lambda_{m}^{2}\right) \int_{-\alpha}^{\alpha} F_{m}(x) \bar{v}_{m}(x)+\sum_{m=0}^{\infty} \int_{-\alpha}^{\alpha} \partial_{x} F_{m}(x) \partial_{x} \bar{v}_{m}(x) .
\end{aligned}
$$

In what follows, we will use the following notation:

$$
\langle\widetilde{F}, v\rangle_{\mathcal{O}}:=\langle\widetilde{F}, v\rangle_{\widetilde{H}^{-1}(\mathcal{O}), H^{1}(\mathcal{O})} .
$$

Remark 2.2. With an obvious abuse of notation, we use $u_{0}, u_{1}$ for the initial conditions in (2.2), and $u_{m}, m \geq 0$, for the coefficients of the decomposition (2.5). 


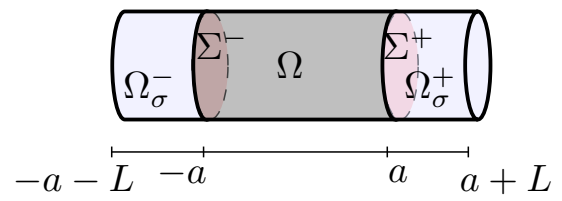

FIG. 2.1. An illustration to the geometric configuration described in Section 2.2 (the domain $\Omega_{c}$ ).

2.2 PML system. Because the domain $\Omega_{\infty}$ is unbounded, to perform simulations, we will truncate the computational domain with the help of the PMLs in the $x$-direction. Recall that the physical domain (the domain of interest), is denoted by

$$
\Omega=I \times \mathcal{S}, \quad I=(-a, a) .
$$

Since the data are supported inside $\Omega$, see (2.1), we can apply the PMLs outside of $\Omega$. The PML layer is used when $|x| \geq a$ and is supposed to be of length $L$ on both sides of $\Omega$. In other words the computational domain (hence the index $c$ in $\Omega_{c}$ ) is

$$
\Omega_{c}:=I_{c} \times \mathcal{S}, \quad I_{c}=(-L-a, L+a) .
$$

The PML domain is then denoted by

$$
\Omega_{\sigma}:=\Omega_{\sigma}^{-} \cup \Omega_{\sigma}^{+}, \quad \Omega_{\sigma}^{-}=(-L-a,-a) \times \mathcal{S}, \quad \Omega_{\sigma}^{+}=(a, L+a) \times \mathcal{S} .
$$

The common interface between $\Omega$ and $\Omega_{\sigma}^{+}$(resp. $\Omega_{\sigma}^{-}$) is denoted by $\Sigma^{+}\left(\right.$resp. $\Sigma^{-}$).

We will use the Bérenger's PMLs, which correspond to a change of variables in the frequency domain. To describe it, let us recall the definition of the Laplace transform for sufficiently regular causal (vanishing on $(-\infty, 0)$ ) functions of polynomial growth:

$$
\hat{v}(s):=\mathcal{L} v=\int_{0}^{\infty} v(t) \mathrm{e}^{-s t} d t, \quad s \in \mathbb{C}^{+}:=\{z \in \mathbb{C}: \operatorname{Re} z>0\} .
$$

This definition extends to causal tempered vector-valued distributions [30, Ch. XVI].

Remark 2.3. In what follows, we will use the following convention: for $s \in \mathbb{C}$, we write $s=s_{r}+i s_{i}, s_{r}, s_{i} \in \mathbb{R}$. Moreover, the square root $\sqrt{s}$ is defined so that its branch cut is $\mathbb{R}_{-}=(-\infty, 0]$ and $\operatorname{Re} \sqrt{s}>0$ for all $s \in \mathbb{C} \backslash(-\infty, 0]$.

The Bérenger's PML then corresponds to the frequency-dependent change of variables:

$$
\tilde{x}= \begin{cases}x+\frac{1}{s} \int_{-a}^{x} \sigma\left(x^{\prime}\right) d x^{\prime}, & x \leq-a, \\ x, & |x|<a, \\ x+\frac{1}{s} \int_{a}^{x} \sigma\left(x^{\prime}\right) d x^{\prime}, & x \geq a .\end{cases}
$$

Here $\sigma(x)$ is a PML damping function that satisfies the following assumption.

Assumption 2 (Damping function).
1) $\sigma \in L^{\infty}\left(I_{c}\right)$;
2) $\sigma \geq 0$ a.e.;
3) $\sigma(x) \equiv 0$ for $|x|<a$;
4) $\sigma(-x)=\sigma(x)$. 
Application of the PML change of variables to (2.2) (where we extend all the functions by zero to $\mathbb{R}_{-}$) and truncation of the computational domain to $\Omega_{c}$ results in the following problem written in the Laplace domain:

$$
\begin{aligned}
& s^{2}\left(1+\frac{\sigma}{s}\right) \hat{u}^{\sigma}-\partial_{x}\left(1+\frac{\sigma}{s}\right)^{-1} \partial_{x} \hat{u}^{\sigma}-\left(1+\frac{\sigma}{s}\right) \Delta_{\perp} \hat{u}^{\sigma}=\hat{f}_{s} \text { in } \Omega_{c} \\
& \hat{f}_{s}=\left(1+\frac{\sigma}{s}\right)\left(\hat{f}+u_{1}+s u_{0}\right) .
\end{aligned}
$$

At the border of $\Omega_{c}$ we equip the resulting system with the Neumann BCs, i.e.

$$
\gamma_{1} \hat{u}^{\sigma}=0, \text { on } \partial \Omega_{c},
$$

where $\gamma_{1}$ is the conormal derivative associated with (2.9). In the strong form, defining

$$
\nabla_{\sigma} v:=\left(\left(1+\frac{\sigma}{s}\right)^{-1} \partial_{x} v,\left(1+\frac{\sigma}{s}\right) \partial_{y} v,\left(1+\frac{\sigma}{s}\right) \partial_{z} v\right)^{t}
$$

we can write the conormal derivative as $\gamma_{1} v=\nabla_{\sigma} v \cdot \boldsymbol{\nu}$.

In the time domain, the resulting system can be written in various ways. We will work with the second order Grote-Sim formulation [36, 7], but most of the results will hold true for other PML formulations (even the first order). The PML system thus reads: find $u^{\sigma}: \mathbb{R}_{+} \times \Omega_{c} \rightarrow \mathbb{R}, \phi=\left(\phi_{x}, \phi_{y}, \phi_{z}\right)^{t}: \mathbb{R}_{+} \times \Omega_{c} \rightarrow \mathbb{R}^{3}$ that satisfy

$$
\begin{aligned}
& \partial_{t}^{2} u^{\sigma}+\sigma \partial_{t} u^{\sigma}-\Delta u^{\sigma}-\operatorname{div} \phi=f, \\
& \partial_{t} \phi_{x}+\sigma \phi_{x}+\sigma \partial_{x} u^{\sigma}=0, \\
& \partial_{t} \phi_{y}=\sigma \partial_{y} u^{\sigma}, \\
& \partial_{t} \phi_{z}=\sigma \partial_{z} u^{\sigma}, \\
& \partial_{\nu} u+\phi \cdot \nu=0 \text { on } \partial \Omega_{c}, \\
& \left.u^{\sigma}\right|_{t=0}=u_{0},\left.\quad \partial_{t} u^{\sigma}\right|_{t=0}=u_{1},\left.\quad \phi\right|_{t=0}=0 .
\end{aligned}
$$

Remark 2.4. In practice, $\phi$ is defined only on $\Omega_{\sigma}$, however, for simplicity of presentation, we defined it on the whole domain $\Omega_{c}$. It is easy to verify that the initial conditions imply that $\phi=0$ inside the physical domain $\Omega$.

The main objective of this article is to quantify the convergence of the solution of (2.10) inside the physical domain $\Omega$ to the solution of (2.2), more precisely,

$$
\left\|u^{\sigma}-u\right\|_{L^{2}\left(0, T ; L^{2}(\Omega)\right)} \rightarrow 0, \quad \text { as } \quad \int_{-a-L}^{-a} \sigma(x) d x+\int_{a}^{a+L} \sigma(x) d x \rightarrow+\infty .
$$

3 Well-posedness and stability of the PML system (2.10). The main result of this section reads.

Theorem 3.1. Let $u_{0}, u_{1}, f$ satisfy Assumption 1. Then there exists a unique solution $u^{\sigma} \in H^{1}\left(0, T ; H^{1}\left(\Omega_{c}\right)\right)$ to (2.10). This solution satisfies

$$
\begin{aligned}
& \left\|\partial_{t} u^{\sigma}\right\|_{L^{2}\left(0, T ; L^{2}(\Omega)\right)}+\left\|\nabla u^{\sigma}\right\|_{L^{2}\left(0, T ; L^{2}(\Omega)\right)} \lesssim \max \left(1,(a+L)^{-1}\right) \max \left(1, T^{\frac{3}{2}}\right) E_{d}(T), \\
& \left\|\partial_{t} u^{\sigma}\right\|_{L^{2}\left(0, T ; L^{2}\left(\Omega_{\sigma}\right)\right)}+\left\|\nabla u^{\sigma}\right\|_{L^{2}\left(0, T ; L^{2}\left(\Omega_{\sigma}\right)\right)} \lesssim C_{\sigma} \max \left(1, T^{\frac{7}{2}}\right) E_{d}(T),
\end{aligned}
$$

where $C_{\sigma}=\max \left(1, L^{-1}\right) \max \left(1,(a+L)^{-1}\right) \max \left(1,\|\sigma\|_{\infty}\right)$. 
To prove the above result, we proceed in three stages:

1. in Section 3.1, we show the existence and uniqueness of the solutions to (2.10a2.10f), in a class of causal tempered distributions. This will be done using Laplace transform techniques, cf. e.g. Dautray and Lions [30]. In principle, the results of this section allow to prove also the PML stability result, similarly to how it was done for PMLs in [16]. Because we will deduce a more optimal result afterwards, in Section 3.2, using an alternative approach, we omit the less optimal proof;

2. in Section 3.2, we will prove the stability of (2.10a-2.10f) by applying Plancherel estimates to an explicit representation of the solution in the Laplace domain;

3. in Section 3.3 we summarize all the obtained results in the proof of Theorem 3.1.

3.1 Existence and uniqueness. We will look for a solution of (2.10a-2.10f) in a class of distributions $T D(X)$ introduced by F. Sayas [56].

Definition $3.2([56])$. Let $X$ be a Banach space. Then the class $T D(X)$ consists of causal (i.e. vanishing on $(-\infty, 0)) X$-valued distributions, s.t. for each $\Phi \in T D(X)$, there exists a causal continuous function $\phi: \mathbb{R} \rightarrow X$ and constants $C, p, m \geq 0$, s.t.

$$
\sup _{t \in(0, \infty)}\|\phi(t)\| \leq C\left(1+t^{p}\right), \quad \text { and } \Phi=\frac{d^{m} \phi}{d t^{m}} .
$$

The class $T D(X)$ is a subset of causal tempered $X$-valued distributions [58, p.417]. We will look for $u^{\sigma} \in T D\left(H^{1}\left(\Omega_{c}\right)\right), \phi \in T D\left(L^{2}\left(\Omega_{c}\right)\right)$ that satisfy (here we use the same notation $\partial_{t}$ for the weak derivative of $u^{\sigma} \in T D\left(H^{1}\left(\Omega_{c}\right)\right)$ as for the classical derivative of a function $u^{\sigma}: \mathbb{R}^{+} \rightarrow H^{1}\left(\Omega_{c}\right)$ )

$$
\partial_{t}^{2} u^{\sigma}+\sigma \partial_{t} u^{\sigma}-\Delta u^{\sigma}-\operatorname{div} \phi=f+\delta_{0} u_{1}+\delta_{0}^{\prime} u_{0}, \text { and }(2.10 b-2.10 e) .
$$

The main result of this section, stated in Proposition 3.3, concerns the well-posedness. Let us remark that we will use less stringent assumptions on the data, because in particular we will work with a wider (not necessarily $L^{2}$ ) class of solutions.

Proposition 3.3 (Existence and Uniqueness). Let $u_{0}, u_{1} \in L^{2}\left(\Omega_{c}\right)$ and $f \in$ $T D\left(\widetilde{H}^{-1}\left(\Omega_{c}\right)\right)$. Then there exists a unique solution $u^{\sigma} \in T D\left(H^{1}\left(\Omega_{c}\right)\right)$ to (3.1).

The proof of the above is based on the following theorem from [56].

Theorem 3.4 (Propositions 3.1.1, 3.1.2, 3.1.3 in [56]). A function $\Phi: \mathbb{C}^{+} \rightarrow X$ is a Laplace transform of $\phi \in T D(X)$ if and only if two conditions below hold true:

1. $\Phi$ is holomorphic in $\mathbb{C}^{+}$;

2. $\Phi$ satisfies the following bound in $\mathbb{C}^{+}$:

$$
\|\Phi(s)\| \leq|s|^{\mu} C_{\Phi}(\operatorname{Re} s), \quad \mu \in \mathbb{R},
$$

where $C_{\Phi}: \mathbb{R}_{+} \rightarrow \mathbb{R}_{+}$is non-increasing and satisfies, with $m \geq 0$ and $C>0$,

$$
C_{\Phi}(\eta) \leq C \eta^{-m} \text {, for all } \eta \in(0,1] \text {. }
$$

The main idea of the proof of the well-posedness of (3.1) lies thus in rewriting the equations (3.1) in the Laplace domain and showing that the above two conditions hold for $\hat{u}^{\sigma}(s)$ (we will omit the proof for $\hat{\phi}(s)$ because it follows almost immediately from the respective results for $\left.\hat{u}^{\sigma}(s)\right)$. Recall that we can rewrite the system (3.1), by 
eliminating $\phi$, in the following compact form, cf. (2.9),

$$
\begin{aligned}
& s^{2}\left(1+\frac{\sigma}{s}\right) \hat{u}^{\sigma}-\partial_{x}\left(1+\frac{\sigma}{s}\right)^{-1} \partial_{x} \hat{u}^{\sigma}-\left(1+\frac{\sigma}{s}\right) \Delta_{\perp} \hat{u}^{\sigma}=\hat{f}_{s} \quad \text { in } \Omega_{c}, \\
& \gamma_{1} \hat{u}^{\sigma}=0 \quad \text { on } \partial \Omega_{c} .
\end{aligned}
$$

To prove Proposition 3.3, we need two auxiliary results, Propositions 3.5 and 3.7. Let us first reformulate (3.3) in a more general, variational, form: provided $\widetilde{F} \in \widetilde{H}^{-1}\left(\Omega_{c}\right)$, find $\hat{u}_{F}^{\sigma} \in H^{1}\left(\Omega_{c}\right)$, s.t. (see also the notation (2.7) for $\langle., .\rangle_{\Omega_{c}}$ ),

$$
\begin{aligned}
& a\left(\hat{u}_{F}^{\sigma}, v\right)=\langle\widetilde{F}, v\rangle_{\Omega_{c}}, \quad \text { for all } v \in H^{1}\left(\Omega_{c}\right), \\
& a(q, v)=s^{2} \int_{\Omega_{c}}\left(1+\frac{\sigma}{s}\right) q \bar{v}+\int_{\Omega_{c}}\left(1+\frac{\sigma}{s}\right)^{-1} \partial_{x} q \partial_{x} \bar{v}+\int_{\Omega_{c}}\left(1+\frac{\sigma}{s}\right) \nabla_{\perp} q \cdot \nabla_{\perp} \bar{v} .
\end{aligned}
$$

Proposition 3.5 (Well-posedness of (3.4)). For all $s \in \mathbb{C}^{+}$, and all $\widetilde{F} \in$ $\widetilde{H}^{-1}\left(\Omega_{c}\right)$, there exists a unique $\hat{u}_{F}^{\sigma} \in H^{1}\left(\Omega_{c}\right)$ that satisfies (3.4). Moreover,

$$
\left\|\hat{u}_{F}^{\sigma}\right\|_{H^{1}\left(\Omega_{c}\right)} \lesssim|s|^{3} \max \left(1, s_{r}^{-7}\right) \max \left(1,\|\sigma\|_{\infty}^{2}\right)\|\widetilde{F}\|_{H^{-1}\left(\Omega_{c}\right)} .
$$

The proof of Proposition 3.5 relies on the modal decomposition applied to (3.4).

Testing the problem (3.4) with $v(x) \phi_{m}(y, z) \in H^{1}\left(\Omega_{c}\right)$ (where $v \in H^{1}\left(I_{c}\right)$ ), using the decomposition (2.5) and the orthogonality of the eigenmodes (2.4), we obtain the following problem: given $\widetilde{F} \in \widetilde{H}^{-1}\left(\Omega_{c}\right)$, find $\hat{u}_{F, m}^{\sigma} \in H^{1}\left(I_{c}\right)$, s.t.

$$
\begin{aligned}
a_{m}\left(\hat{u}_{F, m}^{\sigma}, v\right) & =\left\langle\widetilde{F}, v \phi_{m}\right\rangle_{\Omega_{c}}, \quad \text { for all } v \in H^{1}\left(I_{c}\right), \text { where } \\
a_{m}(q, v) & =\int_{I_{c}}\left(s^{2}+\lambda_{m}^{2}\right) q \bar{v}+\int_{I_{c}} \sigma\left(s+\frac{\lambda_{m}^{2}}{s}\right) q \bar{v}+\int_{I_{c}}\left(1+\frac{\sigma}{s}\right)^{-1} \partial_{x} q \partial_{x} \bar{v}, \\
\left\langle\widetilde{F}, v \phi_{m}\right\rangle_{\Omega_{c}} & \stackrel{(2.6)}{=}\left(1+\lambda_{m}^{2}\right) \int_{I_{c}} F_{m}(x) \bar{v}(x)+\int_{I_{c}} \partial_{x} F_{m}(x) \partial_{x} \bar{v}(x) .
\end{aligned}
$$

The problem (3.6) rewrites: provided $F_{m} \in H^{1}\left(I_{c}\right)$, find $\hat{u}_{F, m}^{\sigma} \in H^{1}\left(I_{c}\right)$, s.t.

$$
a_{m}\left(\hat{u}_{F, m}^{\sigma}, v\right)=\left(1+\lambda_{m}^{2}\right) \int_{I_{c}} F_{m}(x) \bar{v}(x)+\int_{I_{c}} \partial_{x} F_{m}(x) \partial_{x} \bar{v}(x), \forall v \in H^{1}\left(I_{c}\right) .
$$

The above is well-posed, thanks to the following lemma.

LEMma 3.6 (Coercivity, continuity of $a_{m}$ ). For all $s \in \mathbb{C}^{+}$, the sesquilinear form $a_{m}(.,):. H^{1}\left(I_{c}\right) \times H^{1}\left(I_{c}\right) \rightarrow \mathbb{C}$, defined in (3.7), satisfies for all $v \in H^{1}\left(I_{c}\right)$ :

$$
\left|\operatorname{Re} a_{m}(v, v)\right| \gtrsim|s|^{-1} \min \left(1, s_{r}^{4}\right) \min \left(1,\|\sigma\|_{\infty}^{-2}\right)\|v\|_{H^{1}\left(I_{c}\right)}^{2} .
$$

Also, with some $C_{m}(s)>0,\left|a_{m}(q, v)\right| \leq C_{m}(s)\|q\|_{H^{1}\left(I_{c}\right)}\|v\|_{H^{1}\left(I_{c}\right)}, \forall q, v \in H^{1}\left(I_{c}\right)$.

Proof. See Appendix B.

We can now prove Proposition 3.5. 
Proof of Proposition 3.5. Let us fix $s \in \mathbb{C}^{+}$. Let us remark that each solution of (3.4) satisfies (3.9), however, it is not clear whether the solution whose modal decomposition is given through the solutions of the family of problems (3.9) solves (3.4), in particular, whether it belongs to $H^{1}\left(\Omega_{c}\right)$.

Step 1. Well-posedness of (3.9). By the Lax-Milgram theorem, based on Lemma 3.6, the problem (3.9) is well-posed for all $\lambda_{m} \geq 0$.

Step 2. Uniqueness of the solution to (3.4). Because the solution (3.4) satisfies in particular (3.6), the uniqueness of the solution to (3.4) follows from uniqueness of the solution to each of the variational problems (3.6), $m \in \mathbb{N}$.

Step 3. Existence of the solution to (3.4) and a stability estimate.

We will prove the existence by construction. Provided $\hat{u}_{m, F}^{\sigma}, m \in \mathbb{N}$, solving (3.9), let us show that the quantity defined by

$$
\hat{u}_{F}^{\sigma}=\sum_{m=0}^{\infty} \hat{u}_{F, m}^{\sigma} \phi_{m}
$$

belongs to $H^{1}\left(\Omega_{c}\right)$. In this case $\hat{u}_{F}^{\sigma}$ constructed like in (3.10) will satisfy $(3.4)$, by the modal decomposition (2.5). On the other hand, we will prove that $\hat{u}_{F}^{\sigma} \in H^{1}\left(\Omega_{c}\right)$ by proving the following stability bound:

$$
\left\|\hat{u}_{F}^{\sigma}\right\|_{H^{1}\left(\Omega_{c}\right)} \leq C(s) \mid \widetilde{F} \|_{\widetilde{H}^{-1}\left(\Omega_{c}\right)} .
$$

The proof relies on two auxiliary bounds. First of all, by Lemma 3.6, for all $m \in \mathbb{N}$,

$$
\left\|\hat{u}_{F, m}^{\sigma}\right\|_{H^{1}\left(I_{c}\right)}^{2} \lesssim|s| \max \left(1, s_{r}^{-4}\right) \max \left(1,\|\sigma\|_{\infty}^{2}\right)\left|\left\langle\tilde{F}, \hat{u}_{F, m}^{\sigma} \phi_{m}\right\rangle_{\Omega_{c}}\right| .
$$

One could have tried obtaining an estimate for $\left\|\hat{u}_{F, m}^{\sigma}\right\|_{H^{1}\left(I_{c}\right)}$ directly from the above. But this will not result in the desired continuity estimate (3.11), because of the dependence on $\lambda_{m}^{2}$ in (3.8). It is advantageous to leave (3.12) in its present form.

Next, let us bound $\lambda_{m}^{2}\left\|\hat{u}_{F, m}^{\sigma}\right\|_{L^{2}\left(I_{c}\right)}^{2}$ in terms of $\left|\left\langle\widetilde{F}, \hat{u}_{F, m}^{\sigma} \phi_{m}\right\rangle_{\Omega_{c}}\right|$. For this we rewrite (3.9) taking $v=\hat{u}_{F, m}^{\sigma}$ :

$$
\begin{array}{r}
\lambda_{m}^{2} \int_{I_{c}}\left(1+\frac{\sigma}{s}\right)\left|\hat{u}_{F, m}^{\sigma}\right|^{2}+\int_{I_{c}}\left(1+\frac{\sigma}{s}\right)^{-1}\left|\partial_{x} \hat{u}_{F, m}^{\sigma}\right|^{2}+s \int_{I_{c}} \sigma\left|\hat{u}_{F, m}^{\sigma}\right|^{2} \\
=\left\langle\widetilde{F}, \hat{u}_{F, m}^{\sigma} \phi_{m}\right\rangle_{\Omega_{c}}-s^{2}\left\|\hat{u}_{F, m}^{\sigma}\right\|_{L^{2}\left(I_{c}\right)}^{2}
\end{array}
$$

Taking the real part of both sides and using the positivity of all the terms in the left hand side for $s \in \mathbb{C}^{+}$, see in particular (B.3), we obtain the following bound:

$$
\lambda_{m}^{2}\left\|\hat{u}_{F, m}^{\sigma}\right\|_{L^{2}\left(I_{c}\right)}^{2} \leq\left|\left\langle\widetilde{F}, \hat{u}_{F, m}^{\sigma} \phi_{m}\right\rangle_{\Omega_{c}}\right|+|s|^{2}\left\|\hat{u}_{F, m}^{\sigma}\right\|_{L^{2}\left(I_{c}\right)}^{2} .
$$

To bound $|s|^{2}\left\|\hat{u}_{F, m}^{\sigma}\right\|_{L^{2}\left(I_{c}\right)}^{2}$ in the right hand side we use (3.12). This gives

$$
\lambda_{m}^{2} \int_{I_{c}}\left|\hat{u}_{F, m}^{\sigma}\right|^{2} \lesssim \max \left(1,|s|^{3}\right) \max \left(1, s_{r}^{-4}\right) \max \left(1,\|\sigma\|_{\infty}^{2}\right)\left|\left\langle\tilde{F}, \hat{u}_{F, m}^{\sigma} \phi_{m}\right\rangle_{\Omega_{c}}\right| .
$$

Using $\max \left(1,|s|^{3}\right)=|s|^{3} \max \left(|s|^{-3}, 1\right)$ and $s_{r} \leq|s|$, we obtain

$$
\lambda_{m}^{2} \int_{I_{c}}\left|\hat{u}_{F, m}^{\sigma}\right|^{2} \lesssim|s|^{3} \max \left(1, s_{r}^{-7}\right) \max \left(1,\|\sigma\|_{\infty}^{2}\right)\left|\left\langle\tilde{F}, \hat{u}_{F, m}^{\sigma} \phi_{m}\right\rangle_{\Omega_{c}}\right|
$$


Let us now combine (3.13) and (3.12) into a single bound:

$$
\left\|\hat{u}_{F, m}^{\sigma}\right\|_{H^{1}\left(I_{c}\right)}^{2}+\lambda_{m}^{2} \int_{I_{c}}\left|\hat{u}_{F, m}^{\sigma}\right|^{2} \lesssim|s|^{3} \max \left(1, s_{r}^{-7}\right) \max \left(1,\|\sigma\|_{\infty}^{2}\right)\left|\left\langle\tilde{F}, \hat{u}_{F, m}^{\sigma} \phi_{m}\right\rangle_{\Omega_{c}}\right|,
$$

where we used $|s| \max \left(1, s_{r}^{-4}\right) \leq|s|^{3} \max \left(1,|s|^{-2}\right) \max \left(1, s_{r}^{-4}\right) \leq|s|^{3} \max \left(1, s_{r}^{-7}\right)$. Summing the above in $m \in \mathbb{N}$ yields

$$
\left\|\hat{u}_{F}^{\sigma}\right\|_{H^{1}\left(\Omega_{c}\right)}^{2} \lesssim|s|^{3} \max \left(1, s_{r}^{-7}\right) \max \left(1,\|\sigma\|_{\infty}^{2}\right) \sum_{m=0}^{\infty}\left|\left\langle\tilde{F}, \hat{u}_{F, m}^{\sigma} \phi_{m}\right\rangle_{\Omega_{c}}\right| .
$$

Since

$$
\sum_{m=0}^{\infty}\left|\left\langle\tilde{F}, \hat{u}_{F, m}^{\sigma} \phi_{m}\right\rangle_{\Omega_{c}}\right| \stackrel{(2.6)}{\leq} \sum_{m=0}^{\infty}\left(1+\lambda_{m}^{2}\right) \int_{I_{c}}\left|F_{m}\right|\left|\hat{u}_{F, m}^{\sigma}\right|+\sum_{m=0}^{\infty} \int_{I_{c}}\left|\partial_{x} F_{m}\right|\left|\partial_{x} \hat{u}_{F, m}^{\sigma}\right|,
$$

by applying to the above the Cauchy-Schwarz inequality, we obtain

$$
\sum_{m=0}^{\infty}\left|\left\langle\tilde{F}, \hat{u}_{F, m}^{\sigma} \phi_{m}\right\rangle_{\Omega_{c}}\right| \leq\|F\|_{H^{1}\left(\Omega_{c}\right)}\left\|\hat{u}_{F}^{\sigma}\right\|_{H^{1}\left(\Omega_{c}\right)} \equiv\|\widetilde{F}\|_{H^{-1}\left(\Omega_{c}\right)}\left\|\hat{u}_{F}^{\sigma}\right\|_{H^{1}\left(\Omega_{c}\right)} .
$$

Combining (3.14) with (3.15) shows that $\hat{u}_{F}^{\sigma}$ defined in (3.10) belongs to $H^{1}\left(\Omega_{c}\right)$. Moreover, we get (3.11), as well as the desired bound in the statement of Proposition. Therefore, the problem (3.3) is well-posed for all $s \in \mathbb{C}^{+}$. The next proposition shows that $\hat{u}^{\sigma}=\sum_{m=0}^{\infty} \hat{u}_{m}^{\sigma}(s, x) \phi_{m}(y, z)$ depends on $s$ analytically.

Proposition 3.7 (An analytic dependence of $\hat{u}^{\sigma}$ on $s$ ). Let $u_{0}, u_{1} \in L^{2}\left(\Omega_{c}\right)$, and $f \in T D\left(\widetilde{H}^{-1}\left(\Omega_{c}\right)\right)$. Then the function $\hat{u}^{\sigma}: \mathbb{C}^{+} \rightarrow H^{1}\left(\Omega_{c}\right)$, with $\hat{u}^{\sigma}$ being the solution of (3.3), is holomorphic in $\mathbb{C}^{+}$.

Proof. See Appendix C.

Finally, it remains to prove Proposition 3.3.

Proof of Proposition 3.3. The uniqueness is a corollary of the injectivity of the Laplace transform for causal tempered distributions, and the Laplace-domain wellposedness result of Proposition 3.5. For existence, it suffices to verify that the solution of (3.4) (see Proposition 3.5 for the well-posedness) satisfies the conditions of Theorem 3.4. Condition 1 holds by Proposition 3.7; while the condition 2 holds because of the bound (3.5), and the fact that $\hat{f}_{s}=\mathcal{L} f_{s}$, where $f_{s} \in T D\left(\widetilde{H}^{-1}\left(\Omega_{c}\right)\right)$, and thus itself satisfies the bound (3.2) and is analytic in $\mathbb{C}^{+}$.

Remark 3.8. The bounds (3.5) stated in the Laplace domain can be translated into time-domain continuity bounds for $u^{\sigma}$ or its time-domain primitives, cf. e.g. [56, Sections 3.1-3.2], or the proof of Proposition 3.13. Importantly, these bounds will depend on the final time $T$ only polynomially, which would show the stability of the PML problem. However, as discussed before, this leads to non-optimal results, in particular in terms of the time-regularity, compared to the estimates of Section 3.2.

3.2 Stability. To prove the stability of (2.10), we will find an explicit representation to this problem. For this we will reformulate (3.3) as the wave equation in $\Omega$ equipped with the PML Dirichlet-to-Neumann boundary conditions, and provide an explicit expression to its solution in the Laplace domain.

All over this section, we will assume that $u_{0}, u_{1}, f$ satisfy Assumption 1. 
3.2.1 Reformulated PML system in the Laplace domain. In the case when the data is supported inside the physical domain, the PML system (2.10) can be reformulated as the wave equation in the physical domain $(-a, a) \times \mathcal{S}$ with the PML Dirichlet-to-Neumann boundary conditions. The definition and derivation of the PML DtN map is the subject of the next section.

\subsubsection{DtN map in the Laplace domain}

Definition of the PML DtN. The symbol of the PML DtN operator $\boldsymbol{T}_{\sigma}^{+}$is defined as follows. Given $g \in H^{\frac{1}{2}}\left(\Sigma^{+}\right)$, let $G \in H^{1}\left(\Omega_{\sigma}^{+}\right)$solve

$$
\begin{aligned}
& \left(s^{2}+\sigma s\right) G-\left(1+\frac{\sigma}{s}\right) \Delta_{\perp} G-\partial_{x}\left(1+\frac{\sigma}{s}\right)^{-1} G=0 \text { in } \Omega_{\sigma}^{+}, \\
& \left.\gamma_{0} G\right|_{\Sigma^{+}}=g,\left.\quad \gamma_{1} G\right|_{\partial \Omega_{\sigma}^{+} \backslash \Sigma^{+}}=0 .
\end{aligned}
$$

The above problem is well-posed; this is a corollary of Proposition 3.5. We then define

$$
\boldsymbol{T}_{\sigma}^{+} \in \mathcal{L}\left(H^{\frac{1}{2}}\left(\Sigma^{+}\right), \widetilde{H}^{-\frac{1}{2}}\left(\Sigma^{+}\right)\right), \quad \boldsymbol{T}_{\sigma}^{+} g=\left.\gamma_{1} G\right|_{\Sigma^{+}},
$$

where $\widetilde{H}^{-\frac{1}{2}}\left(\Sigma^{+}\right)$is the dual space of $H^{\frac{1}{2}}\left(\Sigma^{+}\right)$. Similarly, we define $\boldsymbol{T}_{\sigma}^{-}$as the DtN map for the domain $\Omega_{\sigma}^{-}$. Let us remark that here the normal in the definition of $\gamma_{1}$ points to the exterior of $\Omega_{\sigma}^{ \pm}$.

DtN map in the Laplace domain: explicit representation. Without loss of generality, let us assume in this section that $\sigma$ is piecewise-continuous. Rewriting (3.16) by using the modal decomposition, we obtain the following ODEs for $m \geq 0$ :

$$
\begin{aligned}
& \left(s^{2}+\lambda_{m}^{2}\right)\left(1+\frac{\sigma}{s}\right) G_{m}-\partial_{x}\left(1+\frac{\sigma}{s}\right)^{-1} \partial_{x} G_{m}=0, \\
& G_{m}(a)=g_{m}, \quad\left(1+\frac{\sigma(a+L)}{s}\right)^{-1} \partial_{x} G_{m}(a+L)=0 .
\end{aligned}
$$

Because for $s \in \mathbb{C}^{+},\left(1+\frac{\sigma(a+L)}{s}\right)^{-1} \neq 0$, cf. (B.10), the last condition in (3.18) is equivalent to $\partial_{x} G_{m}(a+L)=0$. Recall that the above equation is obtained from the equation $\left(s^{2}+\lambda_{m}^{2}\right) v-\partial_{x}^{2} v=0$ by a simple change of variables (2.8). Hence we look for a solution of (3.17) in the following form (cf. Remark 2.3 for the definition of the square root):

$$
G_{m}=C_{g, m}^{+} \mathrm{e}^{\sqrt{s^{2}+\lambda_{m}^{2}} \tilde{x}(x)}+C_{g, m}^{-} \mathrm{e}^{-\sqrt{s^{2}+\lambda_{m}^{2}} \tilde{x}(x)}, \quad \tilde{x}(x)=x+\frac{1}{s} \int_{a}^{x} \sigma\left(x^{\prime}\right) d x^{\prime} .
$$

The coefficients $C_{g, m}^{ \pm}$can be computed from (3.18). Let us denote for brevity

$$
s_{m}:=\sqrt{s^{2}+\lambda_{m}^{2}} .
$$

Then $C_{g, m}^{+}, C_{g, m}^{-}$solve

$$
\left(\begin{array}{cc}
\mathrm{e}^{s_{m} \tilde{x}(a)} & \mathrm{e}^{-s_{m} \tilde{x}(a)} \\
s_{m} \mathrm{e}^{s_{m} \tilde{x}(a+L)} & -s_{m} \mathrm{e}^{-s_{m} \tilde{x}(a+L)}
\end{array}\right)\left(\begin{array}{c}
C_{g, m}^{+} \\
C_{g, m}^{-}
\end{array}\right)=\left(\begin{array}{c}
g_{m} \\
0
\end{array}\right) .
$$

Let us set

$$
\gamma:=\tilde{x}(a+L)-\tilde{x}(a)=L\left(1+\frac{\bar{\sigma}}{s}\right), \quad \bar{\sigma}=\frac{1}{L} \int_{a}^{a+L} \sigma\left(x^{\prime}\right) d x^{\prime}
$$


A straightforward computation gives

$$
C_{g, m}^{ \pm}=\frac{\mathrm{e}^{\mp s_{m}(a+\gamma) \mathrm{e}^{-s_{m} \gamma}}}{1+\mathrm{e}^{-2 s_{m} \gamma}} g_{m}
$$

From (3.19) we obtain

$$
\begin{aligned}
\partial_{x} G_{m}(a) & =s_{m}\left(1+\frac{\sigma(a+)}{s}\right)\left(C_{g, m}^{+} \mathrm{e}^{s_{m} a}-C_{g, m}^{-} \mathrm{e}^{-s_{m} a}\right) \\
& =-s_{m}\left(1+\frac{\sigma(a+)}{s}\right) \frac{1-\mathrm{e}^{-2 s_{m} \gamma}}{1+\mathrm{e}^{-2 s_{m} \gamma}} g_{m} .
\end{aligned}
$$

Finally, using the modal decomposition and the fact that for sufficiently regular $v$, $\left.\gamma_{1} v\right|_{\Sigma^{+}}=-\left.\left(1+\frac{\sigma(a+)}{s}\right)^{-1} \partial_{x} v\right|_{\Sigma_{+}}$(the minus sign comes from the fact that the normal points to the exterior of the domain $\Omega_{\sigma}^{+}$), we obtain the following expression for the symbol of the PML DtN map:

$$
\boldsymbol{T}_{\sigma}^{+} g=\sum_{m=0}^{\infty} \boldsymbol{T}_{\sigma, m}^{+} g_{m}, \quad \boldsymbol{T}_{\sigma, m}^{+}=s_{m} \frac{1-\mathrm{e}^{-2 s_{m} \gamma}}{1+\mathrm{e}^{-2 s_{m} \gamma}} .
$$

Similarly,

$$
\boldsymbol{T}_{\sigma}^{-} g=\sum_{m=0}^{\infty} \boldsymbol{T}_{\sigma, m}^{-} g_{m}, \quad \boldsymbol{T}_{\sigma, m}^{-}=\boldsymbol{T}_{\sigma, m}^{+}=s_{m} \frac{1-\mathrm{e}^{-2 s_{m} \gamma}}{1+\mathrm{e}^{-2 s_{m} \gamma}} .
$$

Let us remark that despite the fact that the derivation was done for $\sigma$ piecewisecontinuous, the expressions $(3.23,3.24)$ remain valid for $\sigma \in L^{\infty}\left(I_{c}\right)$.

Rewriting the PML DtN in terms of the exact DtN map. We will rewrite the PML $\mathrm{DtN}$ map in a more convenient for us form, by comparing it to the exact DtN map, defined similarly to the DtN of (3.16), however, for the problem (2.2). More precisely, let $\Omega^{+}:=(a,+\infty) \times \mathcal{S}$. Given $g \in H^{\frac{1}{2}}\left(\Sigma^{+}\right)$, let $G \in H^{1}\left(\Omega^{+}\right)$solve

$$
s^{2} G-\Delta G=0, \text { in } \Omega^{+},\left.\quad \gamma_{0} G\right|_{\Sigma^{+}}=g,\left.\quad \gamma_{1} G\right|_{\partial \Omega^{+} \backslash \Sigma^{+}}=0 .
$$

For all $s \in \mathbb{C}^{+}$, the above problem is well-posed. The symbol of the exact $\operatorname{DtN} \boldsymbol{T}^{+}$is:

$$
\boldsymbol{T}^{+} \in \mathcal{L}\left(H^{\frac{1}{2}}\left(\Sigma^{+}\right), \widetilde{H}^{-\frac{1}{2}}\left(\Sigma^{+}\right)\right), \quad \boldsymbol{T}^{+} g=\gamma_{1} G .
$$

Similarly we define $\boldsymbol{T}^{-}$, associated to the domain $(-\infty,-a) \times \mathcal{S}$. It is easy to see that

$$
\boldsymbol{T}^{ \pm} g=\sum_{m=0}^{\infty} \boldsymbol{T}_{m}^{ \pm} g_{m}, \quad \quad \boldsymbol{T}_{m}^{ \pm}=s_{m} .
$$

The error between the PML DtN $\mathbf{T}_{\sigma}^{ \pm}$and the exact $\operatorname{DtN} \mathbf{T}^{ \pm}$then rewrites as follows:

$$
\boldsymbol{E}^{ \pm}:=\boldsymbol{T}_{\sigma}^{ \pm}-\boldsymbol{T}^{ \pm}, \quad \boldsymbol{E}^{ \pm} g \equiv \boldsymbol{E} g=\sum_{m=0}^{\infty} \boldsymbol{E}_{m} g_{m}, \quad \boldsymbol{E}_{m}=-s_{m} \frac{2 \mathrm{e}^{-2 s_{m} \gamma}}{1+\mathrm{e}^{-2 s_{m} \gamma}} .
$$

The error between the DtN operators will be crucial for quantification of the error induced by the perfectly matched layer. 


\subsubsection{Reformulated PML system.}

When the data satisfy Assumption 1, we can rewrite the PML system (3.3) in the following form: find $\hat{u}_{\Omega}^{\sigma} \in H^{1}(\Omega)$ that satisfies

$$
\begin{aligned}
& s^{2} \hat{u}_{\Omega}^{\sigma}-\Delta \hat{u}_{\Omega}^{\sigma}=\hat{f}_{s}, \text { in } H^{1}(\Omega), \\
& \left.\left.\partial_{\boldsymbol{\nu}} \hat{u}_{\Omega}^{\sigma}\right|_{\Sigma^{ \pm}} \equiv \gamma_{1} \hat{u}_{\Omega}^{\sigma}\right|_{\Sigma^{ \pm}}=-\boldsymbol{T}_{\sigma}^{ \pm}\left(\gamma_{0} \hat{u}_{\Omega}^{\sigma}\right),\left.\quad \partial_{\boldsymbol{\nu}} \hat{u}_{\Omega}^{\sigma}\right|_{\partial \Omega \backslash\left(\Sigma^{+} \cup \Sigma^{-}\right)}=0 .
\end{aligned}
$$

The systems (3.26) and (3.3) are equivalent in the following sense.

TheOREM 3.9. Let $\hat{f}_{s}=\hat{f}+s u_{0}+u_{1}$, with $f, u_{0}, u_{1}$ satisfying Assumption 1. Then for all $s \in \mathbb{C}^{+}$, the system (3.26) has a unique solution $\hat{u}_{\Omega}^{\sigma} \in H^{1}(\Omega)$. Moreover, $\left.\hat{u}^{\sigma}\right|_{\Omega}=\hat{u}_{\Omega}^{\sigma}$.

Proof. See Appendix D.

3.2.2 Time-domain estimates for the solution of (3.26).

3.2.2.1 Explicit expression of the solution to (3.26) in the Laplace domain.

We will look for a solution of the PML system (3.26) by rewriting it as a perturbation of the solution $u$ of the original problem (2.2):

$$
\hat{u}_{\Omega}^{\sigma}=\left.\hat{u}\right|_{\Omega}+\hat{e}^{\sigma} .
$$

The error $\hat{e}^{\sigma}$ then satisfies a certain boundary-value problem. The respective boundary conditions are obtained using the relation between the DtN operators (3.25):

$$
\left.\partial_{\boldsymbol{\nu}}\left(\hat{e}^{\sigma}+\hat{u}\right)\right|_{\Sigma_{ \pm}}=-\left.\left(\boldsymbol{T}^{ \pm}+\boldsymbol{E}\right) \gamma_{0}\left(\hat{e}^{\sigma}+\hat{u}\right)\right|_{\Sigma_{ \pm}},
$$

which can be simplified using $\partial_{\boldsymbol{\nu}} \hat{u}=-\boldsymbol{T}^{ \pm} \hat{u}$. Altogether, $\hat{e}^{\sigma}$ solves

$$
\begin{aligned}
& s^{2} \hat{e}^{\sigma}-\Delta \hat{e}^{\sigma}=0 \text { in } \Omega \\
& \left.\partial_{\boldsymbol{\nu}} \hat{e}^{\sigma}\right|_{\Sigma_{ \pm}}=-\left.\left(\boldsymbol{T}^{ \pm}+\boldsymbol{E}\right) \gamma_{0} \hat{e}^{\sigma}\right|_{\Sigma_{ \pm}}-\left.\boldsymbol{E} \gamma_{0} \hat{u}\right|_{\Sigma_{ \pm}},\left.\quad \partial_{\boldsymbol{\nu}} \hat{e}^{\sigma}\right|_{\partial \Omega \backslash\left(\Sigma^{+} \cup \Sigma^{-}\right)}=0 .
\end{aligned}
$$

With the decomposition (2.5), we obtain

$$
\begin{aligned}
& s_{m}^{2} \hat{e}_{m}^{\sigma}-\partial_{x}^{2} \hat{e}_{m}^{\sigma}=0, \quad \text { in }(-a, a), \\
& \partial_{x} \hat{e}_{m}^{\sigma}(s, \pm a)=\mp\left(s_{m}+\boldsymbol{E}_{m}\right) \hat{e}_{m}^{\sigma}(s, \pm a) \mp \boldsymbol{E}_{m} \hat{u}_{m}(s, \pm a) .
\end{aligned}
$$

We look for $\hat{e}_{m}^{\sigma}$ in the following form:

$$
\hat{e}_{m}^{\sigma}(s, x)=c_{m}^{+} \mathrm{e}^{s_{m} x}+c_{m}^{-} \mathrm{e}^{-s_{m} x},
$$

where $c_{m}^{+}, c_{m}^{-}$are to be determined from the boundary conditions:

$$
\left(\begin{array}{cc}
\left(2 s_{m}+\boldsymbol{E}_{m}\right) \mathrm{e}^{s_{m} a} & \boldsymbol{E}_{m} \mathrm{e}^{-s_{m} a} \\
\boldsymbol{E}_{m} \mathrm{e}^{-s_{m} a} & \left(2 s_{m}+\boldsymbol{E}_{m}\right) \mathrm{e}^{s_{m} a}
\end{array}\right)\left(\begin{array}{c}
c_{m}^{+} \\
c_{m}^{-}
\end{array}\right)=\left(\begin{array}{c}
-\boldsymbol{E}_{m} \hat{u}_{m}(s, a) \\
-\boldsymbol{E}_{m} \hat{u}_{m}(s,-a)
\end{array}\right) .
$$

Thus, with

$$
\mathbf{C}_{m}=\frac{\boldsymbol{E}_{m}}{\left(2 s_{m}+\boldsymbol{E}_{m}\right)^{2} \mathrm{e}^{2 s_{m} a}-\boldsymbol{E}_{m}^{2} \mathrm{e}^{-2 s_{m} a}},
$$


the solution of the above system reads:

$$
\begin{aligned}
& c_{m}^{+}=\mathbf{C}_{m}\left(-\left(2 s_{m}+\boldsymbol{E}_{m}\right) \mathrm{e}^{s_{m} a} \hat{u}_{m}(s, a)+\boldsymbol{E}_{m} \mathrm{e}^{-s_{m} a} \hat{u}_{m}(s,-a)\right), \\
& c_{m}^{-}=\mathbf{C}_{m}\left(-\left(2 s_{m}+\boldsymbol{E}_{m}\right) \mathrm{e}^{s_{m} a} \hat{u}_{m}(s,-a)+\boldsymbol{E}_{m} \mathrm{e}^{-s_{m} a} \hat{u}_{m}(s, a)\right) .
\end{aligned}
$$

Let us introduce

$$
\begin{aligned}
\boldsymbol{P}_{m} & :=-\frac{\boldsymbol{E}_{m}\left(2 s_{m}+\boldsymbol{E}_{m}\right)}{\left(2 s_{m}+\boldsymbol{E}_{m}\right)^{2}-\boldsymbol{E}_{m}^{2} \mathrm{e}^{-4 s_{m} a}}, \\
\boldsymbol{R}_{m} & :=\frac{\boldsymbol{E}_{m}^{2}}{\left(2 s_{m}+\boldsymbol{E}_{m}\right)^{2}-\boldsymbol{E}_{m}^{2} \mathrm{e}^{-4 s_{m} a}} .
\end{aligned}
$$

With this notation $\hat{e}_{m}^{\sigma}$ rewrites

$$
\begin{aligned}
\hat{e}_{m}^{\sigma}(s, x) & =\boldsymbol{P}_{m} \mathrm{e}^{s_{m}(x-a)} \hat{u}_{m}(s, a)+\boldsymbol{R}_{m} \mathrm{e}^{-2 s_{m} a} \mathrm{e}^{s_{m}(x-a)} \hat{u}_{m}(s,-a) \\
& +\boldsymbol{P}_{m} \mathrm{e}^{-s_{m}(a+x)} \hat{u}_{m}(s,-a)+\boldsymbol{R}_{m} \mathrm{e}^{-2 s_{m} a} \mathrm{e}^{-s_{m}(a+x)} \hat{u}_{m}(s, a) .
\end{aligned}
$$

The goal of the rest of this section is to obtain stability bounds on the solution $u^{\sigma}$, see Proposition 3.13. We will do this via providing "rough" (i.e. not indicating convergence) bounds for the error $e^{\sigma}$. The reader could wonder why we do not present directly convergence results, since, obviously, stability follows from convergence. However, the current stability section allows us to introduce some ingredients and techniques that will be reused for the proof of convergence. We think that this way of presenting the results is easier to follow.

Because further we will need to estimate the $H^{1}$-norm of $e^{\sigma}(t)$, let us introduce some reference problems, which will simplify the analysis.

Remark 3.10. For estimating $\left\|e^{\sigma}(t)\right\|_{L^{2}(\Omega)}$, it is possible to avoid the introduction of the reference problems.

\subsubsection{Rewriting of the error $\hat{e}^{\sigma}$ via reference problems.}

We remark that (3.30) can be rewritten in a simpler form if one notices that the terms of the type $\mathrm{e}^{ \pm s_{m}(a \pm x)} \hat{u}_{m}(s, \pm a)$ correspond to exact solutions of boundary-value problems posed in half-intervals. Let

$$
I^{+}:=(-a, \infty), \quad I^{-}:=(-\infty, a),
$$

and (where the meaning of indices will be explained later)

$$
\begin{aligned}
& \hat{U}_{m}^{-+}:=\mathrm{e}^{s_{m}(x-a)} \hat{u}_{m}(s, a), \quad \hat{U}_{m}^{--}:=\mathrm{e}^{s_{m}(x-a)} \hat{u}_{m}(s,-a), \\
& \hat{U}_{m}^{+-}:=\mathrm{e}^{-s_{m}(x+a)} \hat{u}_{m}(s,-a), \quad \hat{U}_{m}^{++}:=\mathrm{e}^{-s_{m}(x+a)} \hat{u}_{m}(s, a) .
\end{aligned}
$$

The above quantities solve the following boundary-value problems:

$$
\begin{array}{ll}
s_{m}^{2} \hat{U}_{m}^{-+}-\partial_{x}^{2} \hat{U}_{m}^{-+}=0 \text { in } I^{-}, & \hat{U}_{m}^{-+}(s, a)=\hat{u}_{m}(s, a), \\
s_{m}^{2} \hat{U}_{m}^{--}-\partial_{x}^{2} \hat{U}_{m}^{--}=0 \text { in } I^{-}, & \hat{U}_{m}^{--}(s, a)=\hat{u}_{m}(s,-a), \\
s_{m}^{2} \hat{U}_{m}^{+-}-\partial_{x}^{2} \hat{U}_{m}^{+-}=0 \text { in } I^{+}, & \hat{U}_{m}^{+-}(s,-a)=\hat{u}_{m}(s,-a), \\
s_{m}^{2} \hat{U}_{m}^{++}-\partial_{x}^{2} \hat{U}_{m}^{++}=0 \text { in } I^{+}, & \hat{U}_{m}^{++}(s,-a)=\hat{u}_{m}(s, a) .
\end{array}
$$

In the notation $\hat{U}_{m}^{\mathfrak{f r}}, \mathfrak{f}, \mathfrak{r} \in\{-,+\}$, the first index $\mathfrak{f}$ stands for the fact that the problem is solved in $I^{\mathfrak{f}}$, and $\mathfrak{r}$ is used to show that the corresponding boundary condition (with an obvious abuse of notation) $\operatorname{reads} \hat{U}_{m}^{\mathfrak{f r}}(s,-\mathfrak{f} a)=\hat{u}_{m}(s, \mathfrak{r} a)$. 
With these new notations in particular

$$
\hat{e}_{m}^{\sigma}=\left.\boldsymbol{P}_{m}\left(\hat{U}_{m}^{-+}+\hat{U}_{m}^{+-}\right)\right|_{I}+\left.\boldsymbol{R}_{m} \mathrm{e}^{-2 s_{m} a}\left(\hat{U}_{m}^{++}+\hat{U}_{m}^{--}\right)\right|_{I} .
$$

\subsubsection{Time-domain estimates for reference problems.}

Let us introduce for brevity the set of indices

$$
\Lambda=\{--,-+,+-,++\},
$$

and define (a posteriori one will see that the series below converges in a certain norm):

$$
\hat{U}^{\lambda}(s, x, y, z)=\sum_{m=0}^{\infty} \hat{U}_{m}^{\lambda}(s, x) \phi_{m}(y, z), \quad \lambda \in \Lambda,
$$

where $\hat{U}_{m}^{\lambda}$ solve the problems (3.32a-3.32d).

The goal of this section is to derive the stability estimates for $U^{\lambda}$ in the time domain, which will be useful later in the analysis. Let us define

$$
B^{\mathfrak{f}}=I^{\mathfrak{f}} \times \mathcal{S}, \quad \mathfrak{f} \in\{-,+\} .
$$

From (3.32a-3.32d) it follows that $U^{\lambda}, \lambda \in \Lambda$, are the solutions of the boundary-value problems for the wave equation defined below.

$$
\begin{aligned}
& \partial_{t}^{2} U^{-+}-\Delta U^{-+}=0 \text { in } B^{-}, \\
& \partial_{\nu} U^{-+}=0 \text { on } \partial B^{-} \backslash \Sigma^{+},\left.\quad U^{-+}\right|_{\Sigma^{+}}=\left.u\right|_{\Sigma^{+}} \quad \text { (+zero i.c.), } \\
& \partial_{t}^{2} U^{--}-\Delta U^{--}=0 \text { in } B^{-}, \\
& \partial_{\nu} U^{--}=0 \text { on } \partial B^{-} \backslash \Sigma^{+},\left.\quad U^{--}\right|_{\Sigma^{+}}=\left.u\right|_{\Sigma^{-}} \quad \text { (+zero i.c.), } \\
& \partial_{t}^{2} U^{+-}-\Delta U^{+-}=0 \text { in } B^{+}, \\
& \partial_{\nu} U^{+-}=0 \text { on } \partial B^{+} \backslash \Sigma^{-},\left.\quad U^{+-}\right|_{\Sigma^{-}}=\left.u\right|_{\Sigma^{-}} \quad \text { (+zero i.c.), } \\
& \partial_{t}^{2} U^{++}-\Delta U^{++}=0 \text { in } B^{+}, \\
& \partial_{\nu} U^{++}=0 \text { on } \partial B^{+} \backslash \Sigma^{-},\left.\quad U^{++}\right|_{\Sigma^{-}}=\left.u\right|_{\Sigma^{+}} \quad \text { (+zero i.c.). }
\end{aligned}
$$

The stability estimates for the above problems follow almost immediately from the stability estimates for the original problem (2.2).

TheOREm 3.11. For $u_{0}, u_{1}, f$ satisfying Assumption 1, there exists a unique solution to the problem (3.36a-3.36d)

$$
U^{\mathfrak{f r}} \in C^{1}\left([0, T] ; L^{2}\left(B^{\mathfrak{f}}\right)\right) \cap C\left([0, T] ; H^{1}\left(B^{\mathfrak{f}}\right)\right), \quad \mathfrak{f}, \mathfrak{r} \in\{+,-\} .
$$

Moreover, it satisfies the following bound (c.f. (2.3) for the definition of $E_{d}$ ):

$$
\left\|\partial_{t} U^{\lambda}\right\|_{L^{2}\left(0, T ; L^{2}(\Omega)\right)}+\left\|\nabla U^{\lambda}\right\|_{L^{2}\left(0, T ; L^{2}(\Omega)\right)} \lesssim T^{\frac{1}{2}} E_{d}(T), \quad \lambda \in \Lambda .
$$


Proof. Let us show the respective proofs for (3.36a) and (3.36d). The proof for (3.36b) is the same as for (3.36d), and the proof for (3.36c) mimics the proof of (3.36a).

Derivation of regularity estimates for (3.36a). We rewrite $U^{-+}$as follows:

$$
U^{-+}:=u+E^{-+}
$$

where $E^{-+}$solves

$$
\begin{aligned}
& \partial_{t}^{2} E^{-+}-\Delta E^{-+}=-f \text { in } B^{-} \\
& \partial_{\nu} E^{-+}=0 \text { on } \partial B^{-} \backslash \Sigma^{+},\left.\quad E^{-+}\right|_{\Sigma_{+}}=\left.0\right|_{\Sigma_{+}}, \\
& \left.E^{-+}\right|_{t=0}=-u_{0},\left.\quad \partial_{t} E^{-+}\right|_{t=0}=-u_{1} .
\end{aligned}
$$

Then the desired stability and regularity result for $E^{-+}$follows from the same argument as in the proof of Theorem 2.1.

Derivation of regularity estimates for (3.36d). This case is simpler than the previous case, since it suffices to remark that (here we use Assumption 1)

$$
U^{++}(t, x, y, z)=u(t, x+2 a, y, z),
$$

and the stability estimates follow from the result of Theorem 2.1.

The estimates of Theorem 3.11 will be important in obtaining the stability bounds.

3.2.2.4 Laplace-domain estimates for the symbols $\mathbf{P}_{m}(s)$ and $\mathbf{R}_{m}(s)$.

In this section we will provide useful in the sequel estimates for $\boldsymbol{P}_{m}(s)$ and $\boldsymbol{R}_{m}(s)$. We will first rewrite these two expressions in an easier form by replacing in (3.28) and (3.29) $\boldsymbol{E}_{m}$ by its explicit expression (3.25), namely

$$
\boldsymbol{E}_{m}=-2 s_{m} \frac{\mathrm{e}^{-2 s_{m} \gamma}}{1+\mathrm{e}^{-2 s_{m} \gamma}} .
$$

We then get

$$
\boldsymbol{P}_{m}=\frac{\mathrm{e}^{-2 s_{m} \gamma}}{1-\mathrm{e}^{-4 s_{m} \gamma-4 s_{m} a}}, \quad \boldsymbol{R}_{m}=\frac{\mathrm{e}^{-4 s_{m} \gamma}}{1-\mathrm{e}^{-4 s_{m} \gamma-4 s_{m} a}} .
$$

These functions satisfy the following bounds.

Lemma 3.12. For all $s \in \mathbb{C}_{+}, n \in \mathbb{N}$, with $c_{L}=2 \max \left(1,(a+L)^{-1}\right)$, it holds

$$
\left|\boldsymbol{P}_{m}(s)\right| \leq c_{L} \mathrm{e}^{-2 L \operatorname{Re} s} \max \left(1,(\operatorname{Re} s)^{-1}\right), \quad\left|\boldsymbol{R}_{m}(s)\right| \leq c_{L} \mathrm{e}^{-2 L \operatorname{Re} s} \max \left(1,(\operatorname{Re} s)^{-1}\right) .
$$

Proof. By Lemmas 4.5, 4.4, two terms below are strictly positive in $\mathbb{C}^{+}$:

$$
\operatorname{Re}\left(s_{m} \gamma\right) \equiv \operatorname{Re} s_{m} L+\operatorname{Re} \frac{s_{m}}{s} L \bar{\sigma}>0, \quad \forall s \in \mathbb{C}^{+},
$$

and thus

$$
\left|\boldsymbol{R}_{m}(s)\right| \leq\left|\boldsymbol{P}_{m}(s)\right|, \quad s \in \mathbb{C}^{+} .
$$

It remains to get the bound for $\boldsymbol{P}_{m}(s)$ only.

Step 1. An upper bound for $\mathrm{e}^{-s_{m} \gamma}, \mathrm{e}^{-s_{m} a}$. Let $s \in \mathbb{C}^{+}$. Because

$$
\left|\mathrm{e}^{-s_{m} \gamma}\right|=\mathrm{e}^{-\operatorname{Re} s_{m} L-\operatorname{Re} \frac{s_{m}}{s} L \bar{\sigma}},
$$


it suffices to apply Lemma 4.4 and Lemma 4.5 to the above, which gives

$$
\left|\mathrm{e}^{-s_{m} \gamma}\right| \leq \mathrm{e}^{-L \operatorname{Re} s} \text {. }
$$

For the same reason,

$$
\left|\mathrm{e}^{-s_{m} a}\right| \leq \mathrm{e}^{-a \operatorname{Re} s} .
$$

Step 2. A bound for $\boldsymbol{P}_{m}$ and $\boldsymbol{R}_{m}$. From the above it follows

$$
\left|\boldsymbol{P}_{m}(s)\right| \leq \frac{\mathrm{e}^{-2 L \operatorname{Re} s}}{\mid 1-\mathrm{e}^{-4 s_{m} \gamma-4 s_{m} a \mid}} .
$$

We also have, by $(3.39,3.40)$

$$
\left|1-\mathrm{e}^{-4 s_{m} \gamma-4 s_{m} a}\right| \geq 1-\mathrm{e}^{-4(a+L) \operatorname{Re} s} .
$$

Because for $x>0,1-\mathrm{e}^{-x} \geq \frac{1}{2} \min (\log 2, x)>\frac{1}{2} \min (1, x)$,

$$
\left|1-\mathrm{e}^{-4 s_{m} \gamma-4 s_{m} a}\right|^{-1} \leq 2 \max \left(1,(a+L)^{-1}\right) \max \left(1,(\operatorname{Re} s)^{-1}\right) .
$$

Combining (3.42) and (3.41) gives

$$
\left|\boldsymbol{P}_{m}(s)\right| \leq 2 \mathrm{e}^{-2 L \operatorname{Re} s} \max \left(1,(a+L)^{-1}\right) \max \left(1,(\operatorname{Re} s)^{-1}\right) .
$$

\subsubsection{Useful bounds for $\hat{e}^{\sigma}$ in Laplace domain.}

Based on the expressions (3.27) and (3.30), let us define

$$
\begin{aligned}
& \hat{G}^{\mathfrak{f r}}=\sum_{m=0}^{\infty} \hat{G}_{m}^{\mathfrak{f r}} \phi_{m}, \text { where } \hat{G}_{m}^{\mathfrak{f r}}(s, x):=\mathbf{P}_{m}(s) \hat{U}_{m}^{\mathfrak{f r}}(s, x), \quad \mathfrak{f}=\mathfrak{r} \in\{+,-\}, \\
& \hat{G}^{\mathfrak{f r}}=\sum_{m=0}^{\infty} \hat{G}_{m}^{\mathfrak{f r}} \phi_{m}, \text { where } \hat{G}_{m}^{\mathfrak{f r}}(s, x):=\mathbf{R}_{m}(s) \hat{U}_{m}^{\mathfrak{f r}}(s, x), \quad \mathfrak{f} \neq \mathfrak{r} \in\{+,-\} .
\end{aligned}
$$

Each of the above series converges in $H^{1}(\Omega)$. Indeed, by Lemma 3.12, with $c>0$ defined in the statement of the same lemma, we have the following bound:

$$
\left\|\hat{G}^{\lambda}(s)\right\|_{H^{1}(\Omega)}^{2} \leq c_{L}^{2} \max \left(1, s_{r}^{-2}\right)\left\|\hat{U}^{\lambda}(s)\right\|_{H^{1}(\Omega)}^{2}, \quad s \in \mathbb{C}^{+}, \lambda \in \Lambda .
$$

Like in (3.44), we obtain the bound for $\hat{e}^{\sigma}(s)$, valid for $V=H^{1}(\Omega), L^{2}(\Omega)$ and also for $\|\cdot\|_{V} \equiv|\cdot|_{H^{1}(\Omega)}$ :

$$
\left\|\hat{e}^{\sigma}(s)\right\|_{V}^{2} \lesssim c_{L}^{2} \max \left(1, s_{r}^{-2}\right) \sum_{\lambda \in \Lambda}\left\|\hat{U}^{\lambda}(s)\right\|_{V}^{2}, \quad s \in \mathbb{C}^{+} .
$$

\subsubsection{From Laplace domain to the time domain: time-domain esti-} mates for the solution of (3.26).

Let us first of all recall the Plancherel's identity. Given a Banach space $X$, a distribution $v \in T D(X)$, we have

$$
\int_{0}^{\infty} \mathrm{e}^{-2 \eta t}\|v(t)\|_{X}^{2} d t=\frac{1}{2 \pi i} \int_{\eta+i \mathbb{R}}\|\hat{v}(s)\|_{X}^{2} d s .
$$

The stability bound we aim at proving reads. 
Proposition 3.13. Let $u_{0}, u_{1}, f$ satisfy Assumption 1. The solution of (3.26) $\hat{u}_{\Omega}^{\sigma}$ is the Laplace transform of the distribution $u_{\Omega}^{\sigma} \in T D\left(H^{1}(\Omega)\right)$.

It satisfies the following bound, with $E_{d}$ defined in (2.3),

$$
\left\|\partial_{t} u_{\Omega}^{\sigma}\right\|_{L^{2}\left(0, T ; L^{2}(\Omega)\right)}+\left\|\nabla u_{\Omega}^{\sigma}\right\|_{L^{2}\left(0, T ; L^{2}(\Omega)\right)} \lesssim \max \left(1, T^{\frac{3}{2}}\right) \max \left(1,(a+L)^{-1}\right) E_{d}(T) .
$$

Proof. By Proposition 3.9, $\hat{u}_{\Omega}^{\sigma}=\left.\hat{u}^{\sigma}\right|_{\Omega}$, and thus the respective result follows from the fact that $\hat{u}^{\sigma}$ itself is the Laplace transform of the distribution $T D\left(H^{1}\left(\Omega_{c}\right)\right)$, see also Proposition 3.3 and Theorem 3.4. Let us remark that $\hat{e}^{\sigma}(s)$ is the Laplace transform of a distribution of $T D\left(H^{1}(\Omega)\right)$ as well.

Step 1. A bound for $\left\|\nabla u_{\Omega}^{\sigma}\right\|_{L^{2}\left(0, T ; L^{2}(\Omega)\right)}$. With (3.27), we obtain, for all $T \geq 0$,

$$
\int_{0}^{T} \mathrm{e}^{-2 \eta t}\left\|\nabla u_{\Omega}^{\sigma}(t)\right\|_{L^{2}(\Omega)}^{2} d t \lesssim \int_{0}^{T} \mathrm{e}^{-2 \eta t}\left(\|\nabla u(t)\|_{L^{2}(\Omega)}^{2}+\left\|\nabla e^{\sigma}(t)\right\|_{L^{2}(\Omega)}^{2}\right) d t .
$$

To estimate the term in the right-hand side, let us start with the Plancherel theorem and the following inequality, obtained with the help of (3.45),

$$
\begin{aligned}
\int_{0}^{\infty} \mathrm{e}^{-2 \eta t}\left\|\nabla e^{\sigma}(t)\right\|_{L^{2}(\Omega)}^{2} d t & =\frac{1}{2 \pi i} \int_{\eta+i \mathbb{R}}\left\|\nabla \hat{e}^{\sigma}(s)\right\|_{L^{2}(\Omega)}^{2} d s \\
& \lesssim c_{L}^{2} \max \left(1, \eta^{-2}\right) \sum_{\lambda \in \Lambda}\left(\frac{1}{2 \pi i} \int_{\eta+i \mathbb{R}}\left\|\nabla \hat{U}^{\lambda}(s)\right\|_{L^{2}(\Omega)}^{2} d s\right) .
\end{aligned}
$$

Application of the Plancherel theorem to the right hand side of the above yields

$$
\int_{0}^{\infty} \mathrm{e}^{-2 \eta t}\left\|\nabla e^{\sigma}(t)\right\|_{L^{2}(\Omega)}^{2} d t \lesssim c_{L}^{2} \max \left(1, \eta^{-2}\right) \sum_{\lambda \in \Lambda} \int_{0}^{\infty} \mathrm{e}^{-2 \eta t}\left\|\nabla U^{\lambda}(t)\right\|_{L^{2}(\Omega)}^{2} d t .
$$

Finally, by the classical causality argument (cf. Appendix A), for all $T \geq 0$,

$$
\int_{0}^{T} \mathrm{e}^{-2 \eta t}\left\|\nabla e^{\sigma}(t)\right\|_{L^{2}(\Omega)}^{2} d t \lesssim c_{L}^{2} \max \left(1, \eta^{-2}\right) \sum_{\lambda \in \Lambda} \int_{0}^{T} \mathrm{e}^{-2 \eta t}\left\|\nabla U^{\lambda}(t)\right\|_{L^{2}(\Omega)}^{2} d t .
$$

We combine the above bound with (3.47), where we take $\eta=\frac{1}{T}$, to obtain

$$
\begin{aligned}
\int_{0}^{T} \mathrm{e}^{-\frac{2 t}{T}}\left\|\nabla u_{\Omega}^{\sigma}(t)\right\|_{L^{2}(\Omega)}^{2} d t & \lesssim \int_{0}^{T} \mathrm{e}^{-\frac{2 t}{T}}\left(\|\nabla u(t)\|_{L^{2}(\Omega)}^{2}\right. \\
& \left.+c_{L}^{2} \max \left(1, T^{2}\right) \sum_{\lambda \in \Lambda}\left\|\nabla U^{\lambda}(t)\right\|_{L^{2}(\Omega)}^{2}\right) d t
\end{aligned}
$$

Using the results of Theorem 2.1 and of Theorem 3.11, we get, with $\max \left(1, c_{L}\right) \lesssim c_{L}$,

$$
\left\|\nabla u_{\Omega}^{\sigma}\right\|_{L^{2}\left(0, T ; L^{2}(\Omega)\right)}^{2} \lesssim c_{L}^{2} \max \left(1, T^{3}\right) E_{d}^{2} .
$$


Step 2. A bound for $\left\|\partial_{t} u_{\Omega}^{\sigma}\right\|_{L^{2}\left(0, T ; L^{2}(\Omega)\right)}$. Because $\left.u_{\Omega}^{\sigma}\right|_{t=0}=\left.u(0)\right|_{\Omega}$, we rewrite (3.27) and (3.30) as follows:

$$
\left\|\mathcal{L}\left(\partial_{t} u_{\Omega}^{\sigma}-\left.\partial_{t} u\right|_{\Omega}\right)\right\|_{L^{2}(\Omega)}^{2}=\left\|s \hat{e}^{\sigma}(s)\right\|_{L^{2}(\Omega)}^{2} \stackrel{(3.45)}{\lesssim} c_{L}^{2} \max \left(1, s_{r}^{-2}\right) \sum_{\lambda \in \Lambda}\left\|s \hat{U}^{\lambda}\right\|_{L^{2}(\Omega)}^{2} .
$$

By Plancherel theorem, we obtain, with $\eta>0$,

$$
\int_{0}^{\infty} \mathrm{e}^{-2 \eta t}\left\|\partial_{t} u_{\Omega}^{\sigma}-\left.\partial_{t} u\right|_{\Omega}\right\|_{L^{2}(\Omega)}^{2} d t \lesssim \frac{c_{L}^{2}}{2 \pi i} \max \left(1, \eta^{-2}\right) \sum_{\lambda \in \Lambda} \int_{\eta+i \mathbb{R}}\left\|s \hat{U}^{\lambda}(s)\right\|_{L^{2}(\Omega)}^{2} d s .
$$

Because $U^{\lambda} \in C^{1}\left(0, \infty ; L^{2}(\Omega)\right)$, cf. Theorem 3.11, and $U(0)=0, s \hat{U}^{\lambda}(s)=\mathcal{L}\left(\partial_{t} U^{\lambda}\right)$, where the derivative is understood in the strong sense. By the Plancherel theorem, and the causality argument, cf. Appendix A,

$$
\int_{0}^{T} \mathrm{e}^{-2 \eta t}\left\|\partial_{t} u_{\Omega}^{\sigma}-\left.\partial_{t} u\right|_{\Omega}\right\|_{L^{2}(\Omega)}^{2} d t \lesssim c^{2} \max \left(1, \eta^{-2}\right) \sum_{\lambda \in \Lambda} \int_{0}^{T}\left\|\partial_{t} U^{\lambda}(t)\right\|_{L^{2}(\Omega)}^{2} d t .
$$

Finally, choosing $\eta=\frac{1}{T}$, using the results of Theorems 2.1 and 3.11, we obtain the following bound:

$$
\left\|\partial_{t} u_{\Omega}^{\sigma}\right\|_{L^{2}\left(0, T ; L^{2}(\Omega)\right)}^{2} \lesssim c^{2} \max \left(1, T^{3}\right) E_{d}^{2} .
$$

Combining it with (3.48), we prove the statement of the proposition.

By Theorem 3.9, we deduce that the bounds of Proposition 3.13 hold verbatim for $\left.u^{\sigma}\right|_{\Omega}$. It remains to obtain the bounds for the solution $u^{\sigma}$ inside the absorbing layers.

3.2.3 Time-domain estimates for the solution $u^{\sigma}$ inside the layer $\Omega_{\sigma}$. Results of Proposition 3.13 allow us to bound the solution of the PML system (2.10) inside the physical domain $\Omega$. The goal of this section is to obtain stability estimates on the solution inside the PML layer $\Omega_{\sigma}$. For this we will again use its explicit representation; the techniques are basically the same as in Section 3.2.2. Let us derive an estimate for $\left.u^{\sigma}\right|_{\Omega_{\sigma}^{+}}$. Because the computations are almost verbatim the same for $u^{\sigma}$ in $\Omega_{\sigma}^{-}$, we omit them here. Since $\left.\hat{u}^{\sigma}\right|_{\Omega_{\sigma}^{+}}$satisfies the well-posed problem (3.16) with $g=\gamma_{0} \hat{u}^{\sigma} \equiv \hat{u}_{\Omega}^{\sigma}(s, a)$, we can use the explicit solution (3.19), (3.22):

$$
\left.\hat{u}_{m}^{\sigma}\right|_{\Omega_{\sigma}^{+}}=\left(1+\mathrm{e}^{-2 s_{m} \gamma}\right)^{-1}\left(\mathrm{e}^{-s_{m}(a+2 \gamma)+s_{m} \tilde{x}(x)}+\mathrm{e}^{s_{m}(a-\tilde{x}(x))}\right) \hat{u}_{\Omega, m}^{\sigma}(s, a) .
$$

First of all, let us rewrite the $x$-dependent arguments of exponents above:

$$
\begin{aligned}
& a+2 \gamma-\tilde{x}(x)=(a+L-x)+\gamma+\frac{1}{s} \int_{x}^{a+L} \sigma\left(x^{\prime}\right) d x^{\prime}, \\
& a-\tilde{x}(x)=(a-x)-\frac{1}{s} \int_{a}^{x} \sigma\left(x^{\prime}\right) d x^{\prime} .
\end{aligned}
$$


With the above, we can introduce

$$
\begin{aligned}
& \boldsymbol{R}_{\sigma, m}(s, x):=\left(1+\mathrm{e}^{-2 s_{m} \gamma}\right)^{-1} \mathrm{e}^{-s_{m} \gamma} \exp \left(-\frac{s_{m}}{s} \int_{x}^{a+L} \sigma\left(x^{\prime}\right) d x^{\prime}\right), \\
& \left.\boldsymbol{P}_{\sigma, m}(s, x):=\left(1+\mathrm{e}^{-2 s_{m} \gamma}\right)^{-1} \exp \left(-\frac{s_{m}}{s} \int_{a}^{x} \sigma\left(x^{\prime}\right) d x^{\prime}\right)\right) .
\end{aligned}
$$

Then (3.49) rewrites

$$
\left.\hat{u}_{m}^{\sigma}\right|_{\Omega_{\sigma}^{+}}=\boldsymbol{R}_{\sigma, m}(s, x) \mathrm{e}^{s_{m}(x-a-L)} \hat{u}_{\Omega, m}^{\sigma}(s, a)+\boldsymbol{P}_{\sigma, m}(s, x) \mathrm{e}^{-s_{m}(x-a)} \hat{u}_{\Omega, m}^{\sigma}(s, a) .
$$

Replacing $\hat{u}_{\Omega, m}^{\sigma}(s, a)$ by its explicit expression (3.27) and (3.30) (see Theorem 3.9) results in the following expression for the solution $\hat{u}_{m}^{\sigma}$ :

$$
\begin{aligned}
\left.\hat{u}_{m}^{\sigma}\right|_{\Omega_{\sigma}^{+}} & =\boldsymbol{R}_{\sigma, m}(s, x)\left(\left(1+\boldsymbol{P}_{m}+\boldsymbol{R}_{m} \mathrm{e}^{-4 s_{m} a}\right) \hat{U}_{\sigma, m}^{-+}+\mathrm{e}^{-2 s_{m} a}\left(\boldsymbol{R}_{m}+\boldsymbol{P}_{m}\right) \hat{U}_{\sigma, m}^{--}\right) \\
& +\boldsymbol{P}_{\sigma, m}(s, x)\left(\left(1+\boldsymbol{P}_{m}+\boldsymbol{R}_{m} \mathrm{e}^{-4 s_{m} a}\right) \hat{U}_{\sigma, m}^{++}+\mathrm{e}^{-2 s_{m} a}\left(\boldsymbol{R}_{m}+\boldsymbol{P}_{m}\right) \hat{U}_{\sigma, m}^{++}\right),
\end{aligned}
$$

where

$$
\begin{array}{ll}
\hat{U}_{\sigma, m}^{--}:=\mathrm{e}^{s_{m}(x-a-L)} \hat{u}_{m}(s,-a), & \hat{U}_{\sigma, m}^{-+}:=\mathrm{e}^{s_{m}(x-a-L)} \hat{u}_{m}(s, a), \\
\hat{U}_{\sigma, m}^{++}:=\mathrm{e}^{-s_{m}(x-a)} \hat{u}_{m}(s, a), & \hat{U}_{\sigma, m}^{+-}:=\mathrm{e}^{-s_{m}(x-a)} \hat{u}_{m}(s,-a) .
\end{array}
$$

We recognize in (3.50) the solutions to boundary-value problems for the Helmholtz equation on half-intervals, see also the expressions (3.33) and Section 3.2.2.2. Hence, to obtain a bound for e.g. $\left\|\partial_{t} u^{\sigma}\right\|_{L^{2}\left(0, T ; L^{2}\left(\Omega_{\sigma}^{+}\right)\right)}$, we proceed like in Section 3.2.2:

- use the ideas of Section 3.2.2.3 to estimate $U_{\sigma}^{\lambda}, \lambda \in \Lambda$, in the time domain;

- extend the results of Section 3.2.2.4 to provide bounds on $\left\|\boldsymbol{R}_{\sigma, m}(s, .)\right\|_{L^{\infty}\left(\Omega_{\sigma}^{+}\right)}$, $\left\|\boldsymbol{P}_{\sigma, m}(s, .)\right\|_{L^{\infty}\left(\Omega_{\sigma}^{+}\right)}$, that are uniform in $m$ and $s$ for a fixed $\operatorname{Re} s>0$;

- proceed like in Proposition 3.13.

A bound for $\left\|\nabla u^{\sigma}\right\|_{L^{2}\left(0, T ; L^{2}\left(\Omega_{\sigma}\right)\right)}$ can be obtained in a similar manner (with more care taken when estimating $\left.\partial_{x} \boldsymbol{R}_{\sigma, m}(s, x), \partial_{x} \boldsymbol{P}_{\sigma, m}(s, x)\right)$. As this approach mimics the one from Section 3.2.2, we omit the details here and present the main stability result.

Proposition 3.14 (Stability estimates inside the PMLs). Let $u_{0}, u_{1}, f$ satisfy Assumption 1. Then the solution $u^{\sigma}$ to (3.1) satisfies the following stability bound, with $\Omega_{\sigma}=\Omega_{\sigma}^{+} \cup \Omega_{\sigma}^{-}$, and $C_{P M L}=\max \left(1,\|\sigma\|_{\infty}\right) \max \left(1,(a+L)^{-1}\right) \max \left(1, L^{-1}\right)$,

$$
\left\|\nabla u^{\sigma}\right\|_{L^{2}\left(0, T ; L^{2}\left(\Omega_{\sigma}\right)\right.}+\left\|\partial_{t} u^{\sigma}\right\|_{L^{2}\left(0, T ; L^{2}\left(\Omega_{\sigma}\right)\right.} \lesssim C_{P M L} \max \left(1, T^{3}\right) T^{\frac{1}{2}} E_{d} .
$$

3.3 Proof of Theorem 3.1. The existence and uniqueness result in the wider class $T D\left(H^{1}(\Omega)\right)$ follows from Proposition 3.3. Proposition 3.13 shows the bounds for $u_{\Omega}^{\sigma}$ (and thus for $\left.u^{\sigma}\right|_{\Omega}$, by Theorem 3.9). Proposition 3.14 states the bounds on the solution inside the abosorbing layer $\Omega_{\sigma}$. Combining these bounds leads to the desired statement. 
4 Convergence estimates. In the previous section, the stability was proven based on estimates of $\hat{e}^{\sigma}(s)$ for $s \in \mathbb{C}^{+}$. These results are obviously not sufficient for time-domain convergence estimates. Obtaining those is the subject of the present section. We were able to find two (related) approaches to estimate the error induced by the PMLs:

- Laplace transform inversion and contour deformation. Using the explicit form (3.27) and (3.30), we can use the Bromwich inversion formula to obtain the expression for the error in the time domain:

$$
e^{\sigma}(t)=\frac{1}{2 \pi i} \int_{\eta+i \mathbb{R}} \mathrm{e}^{s t} \sum_{n=0}^{\infty} \hat{e}_{n}^{\sigma}(s) \phi_{n} d s=\frac{1}{2 \pi i} \sum_{n=0}^{\infty} \phi_{n} \int_{\eta+i \mathbb{R}} \mathrm{e}^{s t} \hat{e}_{n}^{\sigma}(s) d s
$$

where $\eta>0$. The main idea is then to deform the integration contour(s) (that depend on $t$ and possibly $n)$ to ensure that $\left|\mathrm{e}^{s t} \hat{e}_{n}^{\sigma}(s)\right|$ is minimized along this contour. This allows to obtain an estimate for $\left\|e^{\sigma}\right\|_{L^{\infty}\left(0, T ; L^{2}(\Omega)\right)}$.

- Plancherel's identity. In some cases, it is possible to use the Plancherel's identity (3.46), if the error $\hat{e}^{\sigma}(s)$ can be controlled for all $s \in\{\eta+i \mathbb{R}\}$, with some (well-chosen) $\eta>0$.

The advantage of the first technique is its flexibility; however, typically, it requires more data regularity. The second technique is not always possible to apply. We nonetheless were able to use it. Compared to the first technique, we obtained somewhat less optimal results (in terms of the constants), but with fewer regularity constraints. For this latter reason we will present the results obtained with the second technique.

TheOREM 4.1 (Error of the PMLs). Let $u_{0}, u_{1}, f$ satisfy Assumption 1. Let $u$ solve (2.2) and $u^{\sigma}$ solve (2.10) for $0<t \leq T$. The error $e^{\sigma}=\left.\left(u^{\sigma}-u\right)\right|_{\Omega}$ satisfies:

- for $T<2 L, e^{\sigma} \equiv 0$;

- for $T \geq 2 L$,

$$
\begin{aligned}
\left\|e^{\sigma}\right\|_{L^{2}\left(0, T ; H^{1}(\Omega)\right)} & \lesssim \max \left(1,(a+L)^{-1}\right) \\
& \times \max \left(1, T^{\frac{3}{2}}\right) \max \left(1, \frac{T^{2}}{\bar{\sigma} L^{2}}\right) \exp \left(-\frac{\bar{\sigma} L^{2}}{T}\right) E_{d}(T),
\end{aligned}
$$

with $E_{d}(T)$ defined in (2.3).

This section is dedicated to the proof of Theorem 4.1. It is organized as follows. In Section 4.1 we will relate the convergence of the PMLs to a supremum of a certain quantity along the contour $\eta+i \mathbb{R}$. In Section 4.2 we derive some auxiliary lemmas that allow to characterize this quantity. Section 4.2.3 is dedicated to the derivation of the (quasi-)optimal parameter $\eta$. Finally, we prove Theorem 4.1 in Section 4.

4.1 An auxiliary result. The proof of Theorem 4.1 is based on the following observation, which links the $L^{2}$-time domain estimates for the error of the PML defined in (3.30) to the behaviour of the function $\boldsymbol{P}_{m}(s)$ (3.37) in the Laplace domain.

Remark 4.2. All over this section, we use the following: given $v \in T D\left(L^{2}(\Omega)\right)$, $\hat{v}_{m}(s)$ is the Laplace transform of a distribution $v_{m} \in T D\left(L^{2}(I)\right)$; moreover, $v(t)=$ $\sum_{m=0}^{\infty} v_{m}(t) \phi_{m}$ (i.e. the Laplace transform and decomposition (2.5) commute).

Lemma 4.3. The following bound holds true for the error $e^{\sigma}=u_{\Omega}^{\sigma}-\left.u\right|_{\Omega} \equiv$ 
$\left.\left(u^{\sigma}-u\right)\right|_{\Omega}$, with any $\eta>0$ :

$$
\begin{aligned}
\left\|\mathrm{e}^{\sigma}\right\|_{L^{2}\left(0, T ; H^{1}(\Omega)\right)} & \lesssim \max \left(1, T^{\frac{3}{2}}\right) E_{d}(T) \\
& \times \max \left(1, \eta^{-1}\right) \max \left(1,(a+L)^{-1}\right) \exp (A(\eta, T)),
\end{aligned}
$$

where

$$
A(\eta, T)=\sup _{s \in\{\eta+i \mathbb{R}\}} \sup _{m \in \mathbb{N}} A_{m}(s, T), \quad A_{m}(s, T)=\operatorname{Re}\left(s T-2 L s_{m}-2 L \bar{\sigma} \frac{s_{m}}{s}\right),
$$

Proof. We will proof the bound for $\left\|e^{\sigma}\right\|_{L^{2}\left(0, T ; L^{2}(\Omega)\right)}$. The respective bound in the $L^{2}\left(0, T ; H^{1}(\Omega)\right)$-norm can be obtained in the same way.

Step 1. Bounds in terms of $\boldsymbol{P}_{m}(s)$. From (3.33), and the Plancherel identity, we obtain, for any $\eta>0$,

$$
\begin{gathered}
\int_{0}^{\infty} \mathrm{e}^{-2 \eta t}\left\|\mathrm{e}^{\sigma}(t)\right\|_{L^{2}(\Omega)}^{2} d t \leq \frac{1}{2 \pi i} \int_{\eta+i \mathbb{R}} \sum_{m=0}^{\infty}\left|\mathbf{P}_{m}(s)\right|^{2}\left(\left\|\hat{U}_{m}^{-+}(s)\right\|_{L^{2}(I)}^{2}+\left\|\hat{U}_{m}^{+-}(s)\right\|_{L^{2}(I)}^{2}\right) d s \\
+\frac{1}{2 \pi i} \int_{\eta+i \mathbb{R}} \sum_{m=0}^{\infty}\left|\mathbf{R}_{m}(s)\right|^{2}\left(\left\|\hat{U}_{m}^{--}(s)\right\|_{L^{2}(I)}^{2}+\left\|\hat{U}_{m}^{++}(s)\right\|_{L^{2}(I)}^{2}\right) d s .
\end{gathered}
$$

Using (3.38), the above can be rewritten as:

$$
\begin{gathered}
\int_{0}^{\infty} \mathrm{e}^{-2 \eta t}\left\|\mathrm{e}^{\sigma}(t)\right\|_{L^{2}(\Omega)}^{2} d t \lesssim\left(\sup _{s \in\{\eta+i \mathbb{R}\}} \sup _{m}\left|\mathbf{P}_{m}(s)\right|^{2}\right) \frac{1}{2 \pi i} \int_{\eta+i \mathbb{R}} \sum_{\lambda \in \Lambda} \sum_{m=0}^{\infty}\left\|\hat{U}_{m}^{\lambda}(s)\right\|_{L^{2}(I)}^{2} d s \\
=\left(\sup _{s \in\{\eta+i \mathbb{R}\}} \sup _{m}\left|\mathbf{P}_{m}(s)\right|^{2}\right) \frac{1}{2 \pi i} \int_{\eta+i \mathbb{R}} \sum_{\lambda \in \Lambda}\left\|\hat{U}^{\lambda}(s)\right\|_{L^{2}(\Omega)}^{2} d s .
\end{gathered}
$$

With Plancherel's identity and the causality argument (Appendix A), the above yields

$$
\int_{0}^{T} \mathrm{e}^{-2 \eta t}\left\|\mathrm{e}^{\sigma}(t)\right\|_{L^{2}(\Omega)}^{2} d t \lesssim\left(\sup _{s \in\{\eta+i \mathbb{R}\}} \sup _{m}\left|\mathbf{P}_{m}(s)\right|^{2}\right) \int_{0}^{T} \mathrm{e}^{-2 \eta t} \sum_{\lambda \in \Lambda}\left\|U^{\lambda}(t)\right\|_{L^{2}(\Omega)}^{2} d t .
$$

As $\eta>0$, the above gives

$$
\int_{0}^{T}\left\|\mathrm{e}^{\sigma}(t)\right\|_{L^{2}(\Omega)}^{2} d t \lesssim \mathrm{e}^{2 \eta T}\left(\sup _{s \in\{\eta+i \mathbb{R}\}} \sup _{m}\left|\mathbf{P}_{m}(s)\right|^{2}\right) \int_{0}^{T} \sum_{\lambda \in \Lambda}\left\|U^{\lambda}(t)\right\|_{L^{2}(\Omega)}^{2} d t .
$$

It remains to apply to the above the stability result of Theorem 3.11, where we use the bound $\left\|U^{\lambda}\right\|_{L^{2}\left(0, T ; L^{2}(\Omega)\right)}^{2} \leq T^{2}\left\|\partial_{t} U^{\lambda}\right\|_{L^{2}\left(0, T ; L^{2}(\Omega)\right)}^{2}$, valid because $U^{\lambda}(0)=0$. This finally results in the following bound:

$$
\int_{0}^{T}\left\|\mathrm{e}^{\sigma}(t)\right\|_{L^{2}(\Omega)}^{2} d t \lesssim T^{3} E_{d}^{2}(T) \mathrm{e}^{2 \eta T} \sup _{s \in\{\eta+i \mathbb{R}\}} \sup _{m}\left|\mathbf{P}_{m}(s)\right|^{2} .
$$

The reader can verify that $\left\|e^{\sigma}(t)\right\|_{H^{1}(\Omega)}^{2}$ satisfies the same bound as above (up to a constant), but with $T^{3}$ replaced by $T \max \left(1, T^{2}\right)$. 
Step 2. Rewriting $\mathrm{e}^{2 \eta T} \mathbf{P}_{m}(s)$ via $A_{m}(s, T)$. Let us now consider the term (4.4) that controls the error of the PMLs:

$$
\begin{array}{r}
\sup _{m}\left(\mathrm{e}^{2 \eta T} \sup _{s \in\{\eta+i \mathbb{R}\}}\left|\boldsymbol{P}_{m}(s)\right|^{2}\right) \stackrel{(3.37)}{=} \sup _{m}\left(\mathrm{e}^{2 \eta t} \sup _{s \in\{\eta+i \mathbb{R}\}} \frac{\mathrm{e}^{-4 \operatorname{Re}\left(s_{m} \gamma\right)}}{\mid 1-\mathrm{e}^{-4 s_{m} \gamma-\left.4 s_{m} a\right|^{2}}}\right) \\
=\sup _{m} \sup _{s \in\{\eta+i \mathbb{R}\}} \frac{\mathrm{e}^{2 A_{m}(s, T)}}{\left|1-\mathrm{e}^{-4 s_{m} \gamma-4 s_{m} a}\right|^{2}}
\end{array}
$$

where, see $(3.20),(3.21), A_{m}(s, T)$ is like in (4.3). With $(3.42)$, the above rewrites

$$
\begin{aligned}
\sup _{m}\left(\mathrm{e}^{2 \eta T} \sup _{s \in\{\eta+i \mathbb{R}\}}\left|\boldsymbol{P}_{m}(s)\right|^{2}\right) & \leq 4 \max \left(1,(a+L)^{-2}\right) \\
& \times \sup _{m} \sup _{s \in\{\eta+i \mathbb{R}\}} \max \left(1,(\operatorname{Re} s)^{-2}\right) \mathrm{e}^{2 A_{m}(s, T)} \\
& =4 \max \left(1,(a+L)^{-2}\right) \max \left(1, \eta^{-2}\right) \mathrm{e}^{2 A(\eta, T)} .
\end{aligned}
$$

The bound (4.2) follows by combining (4.6) and (4.4).

4.2 Properties of $\operatorname{Re} s_{m}$ and $\operatorname{Re} \frac{s_{m}}{s}$. From Lemma 4.3 it follows that the error of the PML is controlled by the quantity $A(\eta, T)$. Our goal is to choose $\eta$ so that this quantity is minimized. Because $A(\eta, T)$ depends on the behaviour of $\operatorname{Re} s_{m}$, $\operatorname{Re} \frac{s_{m}}{s}$, in this section we provide some useful properties of these quantities.

\subsubsection{Properties of $\operatorname{Re} s_{m}$.}

\subsubsection{Explicit expressions for $\operatorname{Re} s_{m}$.}

First, remark that $s_{m}=\sqrt{s^{2}+\lambda_{m}^{2}}$ is analytic in $\mathbb{C}^{+}$. Moreover, $s_{m}^{2}=s^{2}+\lambda_{m}^{2}$ implies that

$$
\left(\operatorname{Re} s_{m}\right)^{2}-\left(\operatorname{Im} s_{m}\right)^{2}=s_{r}^{2}-s_{i}^{2}+\lambda_{m}^{2}, \quad \operatorname{Re} s_{m} \operatorname{Im} s_{m}=s_{r} s_{i}
$$

and we obtain the following expression for $\operatorname{Re} s_{m}$ :

$$
\left(\operatorname{Re} s_{m}\right)^{2}=\frac{s_{r}^{2}-s_{i}^{2}+\lambda_{m}^{2}+\sqrt{\Delta}}{2}, \quad \Delta=\left(s_{r}^{2}-s_{i}^{2}+\lambda_{m}^{2}\right)^{2}+4 s_{r}^{2} s_{i}^{2}
$$

Let us rewrite $\Delta$ in a more convenient form:

$$
\begin{aligned}
\Delta & =\left(s_{r}^{2}-s_{i}^{2}\right)^{2}+\lambda_{m}^{4}+2\left(s_{r}^{2}-s_{i}^{2}\right) \lambda_{m}^{2}+4 s_{r}^{2} s_{i}^{2} \\
& =\left(s_{r}^{2}+s_{i}^{2}\right)^{2}+\lambda_{m}^{4}-2\left(s_{r}^{2}+s_{i}^{2}\right) \lambda_{m}^{2}+4 \lambda_{m}^{2} s_{r}^{2}=\left(s_{i}^{2}+s_{r}^{2}-\lambda_{m}^{2}\right)^{2}+4 \lambda_{m}^{2} s_{r}^{2} .
\end{aligned}
$$

We will also need the following simple expression which follows from the above. For $s_{r}^{2}+s_{i}^{2}=\alpha^{2}=$ const, and $0 \leq s_{r} \leq \alpha$, we rewrite it in the simple form:

$$
\left(\operatorname{Re} s_{m}\right)^{2}=s_{r}^{2}+\frac{\lambda_{m}^{2}-\alpha^{2}+\sqrt{\Delta}}{2}, \quad \Delta=\left(\alpha^{2}-\lambda_{m}^{2}\right)^{2}+4 \lambda_{m}^{2} s_{r}^{2}
$$

\subsubsection{Lower bound for $\operatorname{Re} s_{m}$.}

We can show the following result.

LEMma 4.4 (Lower bound for $\operatorname{Re} s_{m}$ ). For all $s \in \mathbb{C}^{+}$, Re $s_{m} \geq \operatorname{Re} s$. 
Proof. We start with (4.9):

$$
\left(\operatorname{Re} s_{m}\right)^{2}-s_{r}^{2}=\frac{\lambda_{m}^{2}-\left(s_{r}^{2}+s_{i}^{2}\right)+\sqrt{\Delta}}{2} .
$$

From (4.8) it follows that the right-hand side in (4.10) is non-negative. Because $\operatorname{Re} s_{m}>0$ (the choice of the branch of $\sqrt{ }$ ), we deduce that $\operatorname{Re} s_{m} \geq \operatorname{Re} s$.

\subsubsection{Properties of $\operatorname{Re} \frac{s_{m}}{s}$.}

\subsubsection{Explicit expressions for $\operatorname{Re} \frac{s_{m}}{s}$.}

The function $\operatorname{Re} \frac{s_{m}}{s}$ is analytic in $\mathbb{C}^{+}$. Like before, let us now rewrite $\operatorname{Re} \frac{s_{m}}{s}$ in a more convenient form.

$$
\begin{aligned}
& \left(\frac{s_{m}}{s}\right)^{2}=\frac{s^{2}+\lambda_{m}^{2}}{s^{2}} \Longrightarrow\left(\operatorname{Re} \frac{s_{m}}{s}\right)^{2}-\left(\operatorname{Im} \frac{s_{m}}{s}\right)^{2}=\frac{|s|^{4}+\lambda_{m}^{2}\left(s_{r}^{2}-s_{i}^{2}\right)}{|s|^{4}} \\
& \left(\operatorname{Re} \frac{s_{m}}{s}\right)\left(\operatorname{Im} \frac{s_{m}}{s}\right)=-\frac{\lambda_{m}^{2}}{|s|^{4}} s_{r} s_{i}
\end{aligned}
$$

and thus we get the following identity

$$
\begin{aligned}
& \left(\operatorname{Re} \frac{s_{m}}{s}\right)^{2}=\frac{|s|^{4}+\lambda_{m}^{2}\left(s_{r}^{2}-s_{i}^{2}\right)+\sqrt{D}}{2|s|^{4}}, \\
& D=\left(|s|^{4}+\lambda_{m}^{2}\left(s_{r}^{2}-s_{i}^{2}\right)\right)^{2}+4 s_{r}^{2} s_{i}^{2} \lambda_{m}^{4} .
\end{aligned}
$$

The expression $D$ can be rewritten with the help of $\Delta$ defined in (4.8):

$$
\begin{aligned}
D & =|s|^{8}+2 \lambda_{m}^{2}\left(s_{r}^{2}-s_{i}^{2}\right)|s|^{4}+\lambda_{m}^{4}\left(s_{r}^{2}+s_{i}^{2}\right)^{2} \\
& =|s|^{4}\left(|s|^{4}-2 \lambda_{m}^{2}\left(s_{r}^{2}+s_{i}^{2}\right)+4 \lambda_{m}^{2} s_{r}^{2}+\lambda_{m}^{4}\right) \\
& =|s|^{4}\left(\left(s_{i}^{2}+s_{r}^{2}-\lambda_{m}^{2}\right)^{2}+4 \lambda_{m}^{2} s_{r}^{2}\right)=|s|^{4} \Delta .
\end{aligned}
$$

We will need the following simple expression for (4.11), for $|s|=\alpha=$ const:

$$
\left(\operatorname{Re} \frac{s_{m}}{s}\right)^{2}=\frac{\alpha^{4}-\lambda_{m}^{2} \alpha^{2}+2 \lambda_{m}^{2} s_{r}^{2}+\alpha^{2} \sqrt{\Delta}}{2 \alpha^{4}}, \quad \Delta=\left(\alpha^{2}-\lambda_{m}^{2}\right)^{2}+4 \lambda_{m}^{2} s_{r}^{2} .
$$

\subsubsection{Positivity of $\operatorname{Re} \frac{s_{m}}{s}$.}

Lemma 4.5 (Positivity of $\operatorname{Re} \frac{s_{m}}{s}$ ). For $s \in \mathbb{C}^{+}, \operatorname{Re} \frac{s_{m}}{s}>0$.

Proof. We remark that

$$
\operatorname{Re} \frac{s_{m}}{s}=\operatorname{Re} \frac{s_{m} \bar{s}}{|s|^{2}}=\frac{s_{r} \operatorname{Re} s_{m}+s_{i} \operatorname{Im} s_{m}}{|s|^{2}} \stackrel{(4.7)}{=} \frac{s_{r} \operatorname{Re} s_{m}+s_{i}^{2} s_{r}\left(\operatorname{Re} s_{m}\right)^{-1}}{|s|^{2}},
$$

which is strictly positive for $s \in \mathbb{C}^{+}$by Lemma 4.4.

4.2.3 A choice of $\eta$ minimizing (4.5). Let us consider (4.3), i.e.

$$
A_{m}(s, T)=\operatorname{Re}\left(s T-2 L s_{m}-2 L \bar{\sigma} \frac{s_{m}}{s}\right) .
$$

We are going to look for $\eta>0$ which would ensure that

$$
A_{m}(s, T)<-\mathrm{C}(\bar{\sigma}, L, T)<0, \quad \text { for all } s \in\{\eta+i \mathbb{R}\}, m \in \mathbb{N} .
$$


For the above to hold true, it is sufficient that

$$
2 L\left(\operatorname{Re} s_{m}+\bar{\sigma} \operatorname{Re} \frac{s_{m}}{s}\right) \geq 2 \eta T, \quad \text { for all } s \in\{\eta+i \mathbb{R}\}, m \in \mathbb{N} .
$$

In this case we would have the following :

$$
(4.14) \Longrightarrow A(\eta, T) \equiv \sup _{m} \sup _{s \in\{\eta+i \mathbb{R}\}} A_{m}(s, T) \leq-\eta T \text {. }
$$

Let us now rewrite (4.14) in a more convenient form. This is an expression we are going to work with. We start with the following technical lemma.

Lemma 4.6. Let $\frac{2 L}{T} \leq 1$. For all $\eta \leq \frac{4 L^{2} \bar{\sigma}}{T^{2}}\left(1+\frac{16 L^{2}}{T^{2}}\right)^{-1}$, the inequality (4.14) holds true.

Proof. Instead of inserting $\eta$ as defined in the statement of the lemma into (4.14) and proving the corresponding result, we would rather derive the bound for $\eta$ stated in the lemma, by starting with (4.14) and showing how it leads to the statement of the lemma.

We start by rewriting (4.14) using the explicit expressions for $\operatorname{Re} s_{m}$ (4.9) and $\operatorname{Re} \frac{s_{m}}{s}$ (4.12), obtained for $|s|=\alpha$ and $s_{r}=\eta>0$,

$$
\begin{aligned}
& \left(\lambda_{m}^{2}-\alpha^{2}+2 \eta^{2}+\sqrt{\Delta}\right)^{\frac{1}{2}}+\frac{\bar{\sigma}}{\alpha^{2}}\left(\alpha^{2}\left(\alpha^{2}-\lambda_{m}^{2}\right)+2 \lambda_{m}^{2} \eta^{2}+\alpha^{2} \sqrt{\Delta}\right)^{\frac{1}{2}} \\
& \geq \frac{\sqrt{2} \eta T}{L}, \quad \Delta=\left(\alpha^{2}-\lambda_{m}^{2}\right)^{2}+4 \lambda_{m}^{2} \eta^{2} .
\end{aligned}
$$

Our goal is to choose $\eta>0$, so that the above inequality holds for all $m \in \mathbb{N}$ and all $\alpha \geq \eta$. We will further simplify our considerations by remarking that for (4.16) to hold true it is sufficient that $\eta$ is s.t.

$$
\left(\lambda_{m}^{2}-\alpha^{2}+\sqrt{\Delta}\right)^{\frac{1}{2}}+\frac{\bar{\sigma}}{\alpha^{2}}\left(\alpha^{2}\left(\alpha^{2}-\lambda_{m}^{2}\right)+\alpha^{2} \sqrt{\Delta}\right)^{\frac{1}{2}} \geq \frac{\sqrt{2} \eta T}{L} .
$$

We consider several cases, where we will (essentially) study $\alpha>\lambda_{m}$ or $\alpha<\lambda_{m}{ }^{1}$

Case 1. $\lambda_{m} \neq 0$. Let $\alpha_{m}:=\xi \lambda_{m}$ with $\xi>1$ be fixed; the actual value of $\xi$ will be determined further. We will consider two cases.

Case 1.1. Choice of $\eta$ when $\alpha \leq \alpha_{m}$. Applying the Young's inequality $x^{2}+y^{2}>$ $2 x y$ to the two terms in the left-hand side of (4.17) shows that for (4.17) to hold true, it is sufficient that for all $0 \leq \alpha \leq \alpha_{m}$,

$$
2 \bar{\sigma}^{\frac{1}{2}} \alpha^{-1}\left(\lambda_{m}^{2}-\alpha^{2}+\sqrt{\Delta}\right)^{\frac{1}{4}}\left(\alpha^{2}\left(\alpha^{2}-\lambda_{m}^{2}\right)+\alpha^{2} \sqrt{\Delta}\right)^{\frac{1}{4}} \geq \frac{\sqrt{2} \eta T}{L} .
$$

The above rewrites

$$
\bar{\sigma}^{\frac{1}{2}} \alpha^{-\frac{1}{2}}\left(\Delta-\left(\lambda_{m}^{2}-\alpha^{2}\right)^{2}\right)^{\frac{1}{4}} \geq \frac{\eta T}{\sqrt{2} L}, \quad \text { for all } \alpha \leq \alpha_{m} .
$$

\footnotetext{
${ }^{1}$ The idea of this splitting comes from the intuition in the frequency domain: for a fixed frequency $\omega \in \mathbb{R}$, s.t. $s=i \omega$, the solution to the Helmholtz equation can be split into the evanescent (containing modes corresponding to $\left.\lambda_{m}>\omega\right)$ and the oscillatory parts $\left(\lambda_{m}<\omega\right)$. With the PML change of variables (2.8), the oscillatory modes are attenuated by choosing $L \bar{\sigma}$ large, while the evanescent modes are attenuated by taking $L$ sufficiently large.
} 
Replacing $\Delta$ by its explicit expression from (4.16), we obtain

$$
\left(\bar{\sigma} \frac{\lambda_{m}}{\alpha}\right)^{\frac{1}{2}} \geq \frac{\eta^{\frac{1}{2}} T}{2 L}, \quad \text { for all } \alpha \leq \alpha_{m} .
$$

Because the minimum of the left hand side is realized at $\alpha=\alpha_{m}=\xi \lambda_{m}$, the above is equivalent to

$$
\eta \leq \frac{4 L^{2} \bar{\sigma}}{T^{2} \xi} .
$$

Case 1.2. Choice of $\eta$ when $\alpha>\alpha_{m}$. In this case we will neglect the first term in (4.17), remarking that for (4.17) to hold true, it suffices that for all $\alpha>\alpha_{m}$,

$$
\frac{\bar{\sigma}}{\alpha^{2}}\left(\alpha^{2}\left(\alpha^{2}-\lambda_{m}^{2}\right)+\alpha^{2} \sqrt{\Delta}\right)^{\frac{1}{2}} \geq \frac{\sqrt{2} \eta T}{L} .
$$

With $\sqrt{\Delta}>\alpha^{2}-\lambda_{m}^{2}$ we see that (4.16) is ensured if $\eta$ satisfies

$$
\frac{\bar{\sigma}}{\alpha}\left(\alpha^{2}-\lambda_{m}^{2}\right)^{\frac{1}{2}} \geq \frac{\eta T}{L}, \quad \text { for all } \alpha>\alpha_{m} .
$$

Because for $\alpha>\alpha_{m}=\xi \lambda_{m}$,

$$
\alpha^{2}-\lambda_{m}^{2}=\alpha^{2}\left(1-\frac{\lambda_{m}^{2}}{\alpha^{2}}\right)>\alpha^{2}\left(1-\frac{\lambda_{m}^{2}}{\alpha_{m}^{2}}\right)=\alpha^{2}\left(1-\xi^{-2}\right),
$$

for (4.19) to hold true it suffices that

$$
\eta \leq \frac{L \bar{\sigma}}{T}\left(1-\xi^{-2}\right)^{\frac{1}{2}} .
$$

Combining the bounds and choosing $\xi$ and $\eta$ to ensure (4.16) for $\alpha \geq \eta$. Let us combine the two bounds (4.18) and (4.20):

$$
\eta \leq \frac{L \bar{\sigma}}{T} \min \left(\frac{4 L}{T \xi},\left(1-\xi^{-2}\right)^{\frac{1}{2}}\right) .
$$

It remains to choose $\xi>1$. Because the first argument of min is decreases in $\xi$, and the second one increases in $\xi$, the value

$$
\max _{\xi>1} \min \left(\frac{4 L}{T \xi},\left(1-\xi^{-2}\right)^{\frac{1}{2}}\right)
$$

is achieved if there exists $\xi>1$, s.t.

$$
\frac{4 L}{T \xi}=\left(1-\xi^{-2}\right)^{\frac{1}{2}} .
$$

The above is satisfied for $\xi^{2}=1+\frac{16 L^{2}}{T^{2}}$. With this choice, (4.21) rewrites

$$
\eta \leq \bar{\sigma} \frac{4 L^{2}}{T^{2}}\left(1+\frac{16 L^{2}}{T^{2}}\right)^{-\frac{1}{2}}
$$


Case 2. $\lambda_{m} \equiv 0$. Evaluating (4.16) in this case results in the following:

$$
\eta+\bar{\sigma} \geq \frac{T}{L} \eta
$$

For the above to hold true it suffices that

$$
\eta \leq \bar{\sigma}\left(\frac{T}{L}-1\right)^{-1}
$$

A final choice of $\eta$. We choose $\eta$ satisfying both (4.22) and (4.23). If $\frac{L}{T}<1$,

$$
\left(\frac{T}{L}-1\right)^{-1}>\frac{4 L^{2}}{T^{2}}
$$

thus it suffices to choose $\eta$ satisfying (4.22). This proves the statement of the Lemma. One of the choices of $\eta$ is given below.

Corollary 4.7. Let $T \geq 2 L$, and let $\eta=\eta_{*}=\frac{L^{2} \bar{\sigma}}{T^{2}}$. Then

$$
A(\eta, T) \leq-\frac{L^{2} \bar{\sigma}}{T}
$$

Proof. Because $T>2 L$, the quantity $\left(1+\frac{16 L^{2}}{T^{2}}\right)^{-\frac{1}{2}}<5^{-\frac{1}{2}}$, and one of the possible choices of $\eta$ satisfying Lemma 4.6 is

$$
\eta=\eta_{*}=\frac{L^{2} \bar{\sigma}}{T^{2}} .
$$

Then the combination of Lemma 4.6 and (4.15) results in the desired statement.

This corollary allows to get a uniform bound for the quantity that controls the error of the PML, see Lemma 4.3, via the connection (4.5).

4.2.4 Proof of Theorem 4.1. Case $T>2 L$. The result of Theorem 4.1 for $T>2 L$ follows by a trivial combination of the bound (4.2) from Lemma 4.3 and Corollary 4.7, by choosing $\eta=\eta_{*}=\frac{L^{2} \bar{\sigma}}{T^{2}}$.

Case $T \leq 2 L$. We will show that $e^{\sigma}(t) \equiv 0$ for all $t<2 L$; this will be done by proving this result for $e_{m}^{\sigma}(t)$ for all $m \in \mathbb{N}$. By [56, Proposition 3.6.1 and discussion afterwards ], it is sufficient to show that $\mathrm{e}^{2 L s} \hat{e}_{m}^{\sigma}$ (which is the Laplace transform of $\left.e_{m}^{\sigma}(t+2 L)\right)$, is the Laplace transform of a causal $T D(\mathbb{R})$ distribution, i.e. satisfies conditions of Theorem 3.4, and thus $e_{m}^{\sigma}(t)$ vanishes for $t<2 L$.

We will use the decomposition (3.33), and show the above for each of the terms in this decomposition. We start with the first term, while for the rest of the terms the result follows similarly:

$$
\hat{e}_{m, \pm}^{\sigma}(s):=\mathbf{P}_{m} \hat{U}_{m}^{+-} .
$$

The analyticity of $\mathrm{e}^{2 L s} \hat{e}_{m, \pm}^{\sigma}(s)$ in $\mathbb{C}^{+}$being a corollary of analyticity of $\mathbf{P}_{m}$ and $\hat{U}_{m}^{+-}$, it remains to show that (3.2) holds for this quantity. Lemma 3.12 yields:

$$
\left\|\mathrm{e}^{2 L s} \hat{e}_{m, \pm}^{\sigma}(s)\right\|_{H^{1}(I)} \leq c \max \left(1, s_{r}^{-1}\right)\left\|\hat{U}_{m}^{+-}(s)\right\|_{H^{1}(I)},
$$

The right hand side satisfies the bound (3.2), because $U_{m}^{+-} \in T D\left(H^{1}(I)\right)$, and thus the conditions of Theorem 3.4 apply. Thus, for $t<2 L, e_{m, \pm}^{\sigma}(t) \equiv 0$. As discussed before, the proof for the remaining terms in (3.33) mimics the above proof, and hence the conclusion. 
Remark 4.8. The choice of $\eta$ as in the corollary 4.7 is not optimal, however, allows to obtain simpler error expressions. A more optimal choice (especially for larger values of $T$ ) would be, cf. Lemma 4.6,

$$
\eta=\frac{4 L^{2}}{T^{2}} \bar{\sigma}\left(1+\frac{16 L^{2}}{T^{2}}\right)^{-1}
$$

which, by (4.15) gives

$$
A(\eta, T) \leq \frac{4 L^{2}}{T^{2}} \bar{\sigma}\left(1+\frac{16 L^{2}}{T^{2}}\right)^{-1} .
$$

Repeating the arguments of the proof of Theorem 4.1, we obtain a more optimal estimate

$$
\begin{aligned}
\left\|e^{\sigma}\right\|_{L^{2}\left(0, T ; L^{2}(\Omega)\right)} & \lesssim T^{\frac{3}{2}} \max \left(1, \frac{T^{2}}{\bar{\sigma} L^{2}}\right) \max \left(1,(a+L)^{-1}\right) \\
& \times \exp \left(-\frac{4 \bar{\sigma} L^{2}}{T}\left(1+\frac{16 L^{2}}{T^{2}}\right)^{-1}\right) E_{d}(T) .
\end{aligned}
$$

Let us remark that the above estimate is close to the one obtained by Diaz, Joly [32], where the error of the Cartesian PMLs in a half-space is shown to be controlled by $\exp \left(-\frac{4 \bar{\sigma} L^{2}}{T}\right)$. We conjecture that in (4.24) the term $\left(1+\frac{16 L^{2}}{T^{2}}\right)^{-1}$ can be waived.

5 Numerical experiments. We have seen in the proof of Theorem 4.1 that for fixed $T>0$, the error of the PMLs decreases exponentially in $\bar{\sigma} L^{2}$. However, when fixing $\bar{\sigma}, L$, the error deteriorates with time $T$. This is consistent with the behaviour of the classical PMLs in a half-space, see Theorem 4 in [32].

Nonetheless, because it is an upper bound, one could wonder whether it is still optimal in the case of the waveguide. The goal of this section is to provide a numerical confirmation to this fact. For this we consider a particular case of the problem (2.2) with the vanishing source and the initial conditions given by

$$
u(0, \boldsymbol{x})=\phi_{m}(y, z) \mathrm{e}^{-\alpha x^{2}} \mathbb{1}_{|x|<\frac{a}{2}}, \quad \partial_{t} u(0, \boldsymbol{x})=0 .
$$

Evidently, in this case $u(t, \boldsymbol{x})=u_{m}(t, x) \phi_{m}(y, z)$, where, in $(0, T) \times \Omega_{b}$,

$$
\partial_{t}^{2} u_{m}+\lambda_{m}^{2} u_{m}-\partial_{x}^{2} u_{m}=0, \quad u_{m}(0, \boldsymbol{x})=\mathrm{e}^{-\alpha x^{2}} \mathbb{1}_{|x|<\frac{a}{2}}, \quad \partial_{t} u_{m}(0, \boldsymbol{x})=0 .
$$

We then apply the PMLs to the above problem and discretize the resulting equations using the ideas of [7]. Provided a simulation time $T>0$, the solution $u_{m}^{\sigma}(x, t)$, $x \in(-a, a)$, obtained with the help of the PMLs, is then compared to the solution $u_{m}(x, t), x \in(-a, a)$, computed on the domain $\left(-a-\frac{T}{2}, a+\frac{T}{2}\right)$. The size of the latter domain is chosen so that the wave reflected from the boundaries does not reach in time $T$ the physical domain $(-a, a)$. In all the experiments $a=0.5, \alpha=10^{3}$. We also choose the quadratic profile of the damping function:

$$
\sigma(x)=\sigma_{0}(x-|a|)^{2}>0, \quad|x| \geq a .
$$

We then measure the respective (discrete) relative norms

$$
e_{m}^{\bar{\sigma}, L, T}=\left\|u_{m}^{\sigma}-u_{m}\right\|_{L^{2}\left(0, T ; L^{2}(-a, a)\right)} /\left\|u_{m}\right\|_{L^{2}\left(0, T ; L^{2}(-a, a)\right)} .
$$




\begin{tabular}{|c|l|c|}
$i$ & $e_{m}^{\bar{\sigma}, L, T}$ & $d_{i}$ \\
\hline 0 & 0.17 & - \\
1 & 0.052 & 0.31 \\
2 & 0.018 & 0.35 \\
3 & $6.8 \cdot 10^{-3}$ & 0.38 \\
4 & $2.7 \cdot 10^{-3}$ & 0.4 \\
5 & $1.1 \cdot 10^{-3}$ & 0.41 \\
6 & $4.4 \cdot 10^{-4}$ & 0.4 \\
7 & $1.8 \cdot 10^{-4}$ & 0.41 \\
8 & $7.7 \cdot 10^{-5}$ & 0.43 \\
9 & $3.3 \cdot 10^{-5}$ & 0.43 \\
10 & $1.4 \cdot 10^{-5}$ & 0.42
\end{tabular}

\begin{tabular}{|l|c|}
$e_{m}^{\bar{\sigma}, L, T}$ & $d_{i}$ \\
\hline 0.28 & - \\
$1.5 \cdot 10^{-2}$ & 0.05 \\
$2.2 \cdot 10^{-3}$ & 0.14 \\
$3.8 \cdot 10^{-4}$ & 0.17 \\
$7.4 \cdot 10^{-5}$ & 0.19 \\
$1.5 \cdot 10^{-5}$ & 0.21 \\
$3.3 \cdot 10^{-6}$ & 0.22 \\
$7.4 \cdot 10^{-7}$ & 0.22 \\
$1.6 \cdot 10^{-7}$ & 0.23 \\
$3.9 \cdot 10^{-8}$ & 0.23 \\
$9 \cdot 10^{-9}$ & 0.24
\end{tabular}

\begin{tabular}{|l|c|}
$e_{m}^{\bar{\sigma}, L, T}$ & $d_{i}$ \\
\hline $1.2 \cdot 10^{-7}$ & - \\
$4.5 \cdot 10^{-7}$ & 3.7 \\
$1.6 \cdot 10^{-6}$ & 3.6 \\
$5.8 \cdot 10^{-6}$ & 3.6 \\
$2.1 \cdot 10^{-5}$ & 3.6 \\
$7.7 \cdot 10^{-5}$ & 3.6 \\
$2.8 \cdot 10^{-4}$ & 3.7 \\
$1.1 \cdot 10^{-3}$ & 3.8 \\
$4 \cdot 10^{-3}$ & 3.9 \\
$1.7 \cdot 10^{-2}$ & 4.1 \\
$8.2 \cdot 10^{-2}$ & 4.8
\end{tabular}

The data for Experiment 1 (left), Experiment 2 (middle) and Experiment 3 (right) described in Section 5.

By varying one of the values of $\sigma_{0}, L, T$, while keeping the rest of parameters fixed, we verify numerically the convergence rate given by Theorem 4.1 . For this we measure the quantity

$$
d_{i}:=\frac{e_{i+1}}{e_{i}},
$$

where the quantity $e_{i}=\mathrm{e}_{m}^{\bar{\sigma}, L, T}$ measured in the $i$ th experiment. In each of the experiments the parameters are chosen in a way that ensures that the theoretical value of $d_{i}$ remains approximately constant (more precisely, in our estimates of the theoretical value we neglect the terms that depend on $L, T$ polynomially in the estimate of Theorem 4.1). We perform three experiments (where $\lambda_{m}=100$ in all the experiments):

- Experiment 1: $L=0.5, T=10$ and $\bar{\sigma}_{i+1}=\bar{\sigma}_{i}+\Delta \bar{\sigma}$, with $\Delta \bar{\sigma}=5, \bar{\sigma}_{0}=5$ and $\sigma_{10}=55$. In this case, the theoretical value (which we denote by $d_{t h}$ ) of $d_{i}$ for all $i$ is given by

$$
d_{t h}=\exp \left(-\frac{L^{2}}{T}\left(\bar{\sigma}_{i}-\bar{\sigma}_{i-1}\right)\right)=\exp \left(-\frac{L^{2}}{T} \Delta \bar{\sigma}\right) \approx 0.88 .
$$

The measured errors are shown in Table 5.1, left. We observe that in the numerical experiments $d_{i}$ remains almost constant, however, is closer to 0.43 , which indicates that the correct rate is probably closer to $\exp \left(-\gamma \frac{L^{2}}{T} \Delta \bar{\sigma}\right)$, with $\gamma>1$. An explanation to this can be found in Remark 4.8.

- Experiment 2: $\bar{\sigma}=30, T=10$ and $L_{i+1}^{2} \approx L_{i}^{2}+(\Delta L)^{2}$, with $(\Delta L)^{2}=0.1$, $L_{0}=0.1$ and $L_{10}=1.0$. In this case, the theoretical value of $d_{i}$ is given by

$$
d_{t h}=\exp \left(-\frac{\bar{\sigma}}{T}\left(L_{i+1}^{2}-L_{i}^{2}\right)\right)=\exp \left(-\bar{\sigma} \frac{(\Delta L)^{2}}{T}\right) \approx 0.74
$$

The measured errors are shown in Table 5.1, middle. In the numerical experiments $d_{i}$ remains almost constant, however, is closer to 0.23 . This can be explained like in Experiment 1.

- Experiment 3: $\bar{\sigma}=30, L=0.5, T_{i+1}^{-1}=T_{i}^{-1}-(\Delta T)^{-1},(\Delta T)^{-1}=0.025$, $T_{0} \approx 3.6$ and $T_{10}=40$. In this case, the theoretical value of $d_{i}$ is given by

$$
d_{t h}=\exp \left(-L^{2} \bar{\sigma}\left(T_{i+1}^{-1}-T_{i}^{-1}\right)\right)=\exp \left(\bar{\sigma} L^{2}(\Delta T)^{-1}\right) \approx 1.21 .
$$


The measured errors are shown in Table 5.1, right. In the numerical experiments the value of $d_{i}$ is closer to 3.7, which can be explained like in the previous cases. The deterioration of $d_{i}$ for longer times can be attributed the fact that in the convergence estimate of Theorem 4.1 the polynomial time-dependent terms may become significant.

Our experiments confirm the results of Theorem 4.1: the error decreases exponentially with $L^{2} \bar{\sigma}$, and deteriorates exponentially with $\frac{1}{T}, T$ being the simulation time.

6 Conclusions. In this work we have proven convergence and stability of the PMLs in 3D waveguides. Our results are consistent with the ones we found in the literature for the 2D half-space problem [32], in the sense that the error of the PMLs is shown to behave like $\exp \left(-\frac{\bar{\sigma} L^{2}}{T}\right)$, where $L$ is the length of the PML layer, $\bar{\sigma}$ is the average absorption rate and $T$ is the computation time.

The stability bounds and the convergence proof presented in this work rely heavily on the explicit representation of the solution to the PML problem, which is enabled by the structure of the isotropic wave equation in the waveguide. Nonetheless, as indicated in the beginning of Section 3, stability of the perfectly matched layers can be shown by omitting the explicit representation of the solution, by energy-like arguments in the Laplace domain. Let us remark that while the approach in this article was suggested for the waveguides, we think that it can be used in other cases when the perfectly matched layers are used in one of the coordinates (e.g. for the analysis of the radial PMLs [27]). Moreover, we believe that the techniques of the present paper can be extended to the error analysis of the PMLs constructed for the dispersive problems in [15] and [16], for which, up to our knowledge, no information about the PML convergence is available.

Appendix A. Causality. Given a Banach space $X$, let

$$
\begin{aligned}
& L_{\eta}^{2}(\mathbb{R} ; X):=\left\{v \in T D(X): \int_{0}^{\infty} \mathrm{e}^{-2 \eta t}\|v\|_{X}^{2} d t<\infty\right\}, \\
& H_{\eta}^{m}(\mathbb{R} ; X):=\left\{v \in T D(X): \int_{0}^{\infty} \mathrm{e}^{-2 \eta t} \sum_{\alpha=0}^{m}\left\|\partial_{t}^{\alpha} v\right\|_{X}^{2} d t<\infty\right\} .
\end{aligned}
$$

Lemma A.1. Given $m \geq 0, \eta>0, X, Y$ two Banach spaces, let $\mathcal{G}: H_{\eta}^{m}(\mathbb{R} ; X) \rightarrow$ $L_{\eta}^{2}(\mathbb{R} ; Y)$ be a convolution operator, defined in the Laplace domain as follows, with $\mathbb{G} \in T D(\mathcal{L}(X, Y)):$

$$
\mathcal{G} v=\mathcal{L}^{-1}(\hat{\mathbb{G}}(s) \hat{v}(s))=\mathbb{G} * v, \quad v \in H_{\eta}^{m}(\mathbb{R} ; X) .
$$

Assume that $\mathcal{G}$ satisfies, for all $v \in H_{\eta}^{m}(\mathbb{R} ; X)$,

$$
\int_{0}^{\infty} \mathrm{e}^{-2 \eta t}\|\mathcal{G} v\|_{X}^{2} d t \leq C(\eta) \int_{0}^{\infty} \mathrm{e}^{-2 \eta t}\left\|\partial_{t}^{m} v\right\|_{Y}^{2} d t,
$$

where $C$ depends on $\eta$ only. Then, for all $T>0$,

$$
\int_{0}^{T} \mathrm{e}^{-2 \eta t}\|\mathcal{G} v\|_{X}^{2} d t \leq C(\eta) \int_{0}^{T} \mathrm{e}^{-2 \eta t}\left\|\partial_{t}^{m} v\right\|_{Y}^{2} d t .
$$


Proof. Given $v \in H_{\eta}^{m}(\mathbb{R} ; X)$, let us define $v_{T} \in L_{l o c}^{2}(\mathbb{R} ; X)$ as follows:

$$
v_{T}(t)= \begin{cases}v, & t \leq T, \\ 0, & m=0 \text { and } t>T, \\ \sum_{k=0}^{m-1} \frac{(t-T)^{k}}{k !} \partial_{t}^{k} v(T), & m>0 \text { and } t>T .\end{cases}
$$

It is easy to see that $v_{T} \in H_{\eta}^{m}(\mathbb{R} ; X)$. Moreover, $\partial_{t}^{m} v_{T}=\mathbb{1}_{(0, T)} v$. By (A.1),

$$
\int_{0}^{\infty} \mathrm{e}^{-2 \eta t}\left\|\mathcal{G} v_{T}\right\|_{X}^{2} d t \leq C(\eta) \int_{0}^{T} \mathrm{e}^{-2 \eta t}\left\|\partial_{t}^{m} v\right\|_{Y}^{2} d t
$$

Moreover, on $(0, T), g * v_{T}=g * v$ (this can be proven e.g. by showing that $\mathrm{e}^{2 T s}\left(\hat{v}_{T}-\right.$ $\hat{v}) \hat{g}(s)$ satisfies (3.2) and invoking [56, Proposition 3.6.1]). With this observation, (A.3) implies (A.2).

Appendix B. Proof of Lemma 3.6. Step 1. Coercivity. We will consider the following two cases separately: $\lambda_{m}^{2} \geq s_{i}^{2}$ and $\lambda_{m}^{2}<s_{i}^{2}$.

Step 1.2. Case $\lambda_{m}^{2} \geq s_{i}^{2}$. Let us take the real part of $a_{m}(v, v)$ :

$$
\begin{aligned}
\operatorname{Re} a_{m}(v, v) & =\int_{I_{c}}\left(s_{r}^{2}-s_{i}^{2}+\lambda_{m}^{2}\right)|v|^{2}+\int_{I_{c}} \sigma s_{r}\left(1+\frac{\lambda_{m}^{2}}{|s|^{2}}\right)|v|^{2} \\
& +\int_{I_{c}} \operatorname{Re}\left(\frac{s}{s+\sigma}\right)\left|\partial_{x} v\right|^{2} .
\end{aligned}
$$

Because $\lambda_{m}^{2} \geq s_{i}^{2}$, and $s_{r}>0$, the following two inequalities hold:

$$
\int_{I_{c}}\left(s_{r}^{2}-s_{i}^{2}+\lambda_{m}^{2}\right)|v|^{2} \geq s_{r}^{2}\|v\|_{L^{2}\left(I_{c}\right)}^{2}, \quad \int_{I_{c}} \sigma s_{r}\left(1+\frac{\lambda_{m}^{2}}{|s|^{2}}\right)|v|^{2} \geq 0 .
$$

The third term in (B.1) can be bounded from below as follows:

$$
\begin{aligned}
\operatorname{Re}\left(\frac{s}{s+\sigma}\right) & =\frac{|s|^{2}+s_{r} \sigma}{|s+\sigma|^{2}} \geq \frac{|s|^{2}}{2\left(|s|^{2}+\sigma^{2}\right)} \geq \frac{|s|^{2}}{4} \min \left(|s|^{-2}, \sigma^{-2}\right) \\
& \geq \frac{1}{4} \min \left(1,\|\sigma\|_{\infty}^{-2}|s|^{2}\right) \geq \frac{1}{4} \min \left(1,\|\sigma\|_{\infty}^{-2}\right) \min \left(1, s_{r}^{2}\right) .
\end{aligned}
$$

Combining (B.2) and (B.3) into (B.1), we get the following lower bound:

$$
\operatorname{Re} a_{m}(v, v) \geq \frac{1}{4} \min \left(1,\|\sigma\|_{\infty}^{-2}\right) \min \left(1, s_{r}^{2}\right)\|v\|_{H^{1}\left(I_{c}\right)}^{2}
$$

Step 1.2. Case $\lambda_{m}^{2}<s_{i}^{2}$. In this case we compute the real part of $a_{m}(v, s v)$ :

$$
\begin{aligned}
\operatorname{Re} a_{m}(v, s v) & =\int_{I_{c}}\left(|s|^{2} s_{r}+s_{r} \lambda_{m}^{2}\right)|v|^{2}+\int_{I_{c}}\left(|s|^{2}+\operatorname{Re} \frac{\lambda_{m}^{2} \bar{s}}{s}\right) \sigma|v|^{2} \\
& +\int_{I_{c}} \operatorname{Re} \frac{|s|^{2}}{s+\sigma}\left|\partial_{x} v\right|^{2} .
\end{aligned}
$$


In the lower bound for the first term we neglect $\lambda_{m}^{2}|v|^{2}$ :

$$
\int_{I_{c}}\left(|s|^{2} s_{r}+s_{r} \lambda_{m}^{2}\right)|v|^{2} \geq|s|^{2} s_{r}\|v\|_{L^{2}\left(I_{c}\right)}^{2} \geq \min \left(1, s_{r}^{3}\right)\|v\|_{L^{2}\left(I_{c}\right)}^{2} .
$$

The second term in (B.5) is non-negative:

$$
\begin{aligned}
\operatorname{Re}\left(|s|^{2}+\frac{\lambda_{m}^{2} \bar{s}}{s}\right) & =|s|^{2}+\lambda_{m}^{2} \frac{s_{r}^{2}-s_{i}^{2}}{|s|^{2}}=\frac{\left(s_{r}^{2}+s_{i}^{2}\right)^{2}+\lambda_{m}^{2}\left(s_{r}^{2}-s_{i}^{2}\right)}{|s|^{2}} \\
& =\frac{s_{r}^{4}+2 s_{i}^{2} s_{r}^{2}+\lambda_{m}^{2} s_{r}^{2}+s_{i}^{2}\left(s_{i}^{2}-\lambda_{m}^{2}\right)}{|s|^{2}} \geq 0,
\end{aligned}
$$

where the last inequality follows from $\lambda_{m}^{2}<s_{i}^{2}$. It remains to obtain a lower bound for the third term in (B.5):

$$
\begin{aligned}
\operatorname{Re} \frac{|s|^{2}}{s+\sigma} & =\frac{|s|^{2}\left(s_{r}+\sigma\right)}{|s+\sigma|^{2}} \geq \frac{|s|^{2} s_{r}}{2\left(|s|^{2}+\sigma^{2}\right)} \geq \frac{|s|^{2} s_{r}}{4} \min \left(|s|^{-2},\|\sigma\|_{\infty}^{-2}\right) \\
& \geq \frac{s_{r}}{4} \min \left(1,\|\sigma\|_{\infty}^{-2}\right) \min \left(1, s_{r}^{2}\right) \geq \frac{1}{4} \min \left(1,\|\sigma\|_{\infty}^{-2}\right) \min \left(1, s_{r}^{3}\right) .
\end{aligned}
$$

Combining (B.6), (B.7) and (B.8) in (B.5) results in

$$
\operatorname{Re} a_{m}(v, s v) \geq \frac{1}{4} \min \left(1,\|\sigma\|_{\infty}^{-2}\right) \min \left(1, s_{r}^{3}\right)\|v\|_{H^{1}\left(I_{c}\right)}^{2},
$$

or, alternatively,

$$
\left|\operatorname{Re} a_{m}(v, v)\right| \geq \frac{1}{4|s|} \min \left(1,\|\sigma\|_{\infty}^{-2}\right) \min \left(1, s_{r}^{3}\right)\|v\|_{H^{1}\left(I_{c}\right)}^{2},
$$

Step 1.3. A general bound. Combining (B.9) and (B.4), we deduce the desired bound from the statement of the lemma:

$$
\begin{aligned}
\left|\operatorname{Re} a_{m}(v, v)\right| & \geq \frac{1}{4} \min \left(1,|s|^{-1}\right) \min \left(1,\|\sigma\|_{\infty}^{-2}\right) \min \left(1, s_{r}^{3}\right)\|v\|_{H^{1}\left(I_{c}\right)}^{2} \\
& \geq \frac{1}{4}|s|^{-1} \min \left(1,\|\sigma\|_{\infty}^{-2}\right) \min \left(1, s_{r}^{4}\right)\|v\|_{H^{1}\left(I_{c}\right)}^{2},
\end{aligned}
$$

where we used $\min \left(1,|s|^{-1}\right) \geq|s|^{-1} \min (1,|s|) \geq|s|^{-1} \min \left(1, s_{r}\right)$.

Step 2. Continuity. The continuity of $a_{m}(q, v)$ is obvious if one shows that $\left|\left(1+\frac{\sigma}{s}\right)\right|^{-1}$ is bounded for all $s \in \mathbb{C}^{+}$. This is however easy to verify:

$$
\left|\left(1+\frac{\sigma}{s}\right)\right| \geq \operatorname{Re}\left(1+\frac{\sigma}{s}\right)=1+\frac{\sigma \operatorname{Re} s}{|s|^{2}} .
$$

Later on we will need the continuity bound in an explicit form:

$$
\begin{aligned}
\left|a_{m}(q, v)\right| & \leq|s|^{2} \max \left(|s|,\|\sigma\|_{\infty}|s|^{-1}\right)\|q\|_{L^{2}\left(I_{c}\right)}\|v\|_{L^{2}\left(I_{c}\right)} \\
(\text { B.11) } & +\lambda_{m}^{2} \max \left(1,\|\sigma\|_{\infty}|s|^{-1}\right)\|q\|_{L^{2}\left(I_{c}\right)}\|v\|_{L^{2}\left(I_{c}\right)}+|s|^{2}\left\|\partial_{x} q\right\|_{L^{2}\left(I_{c}\right)}\left\|\partial_{x} v\right\|_{L^{2}\left(I_{c}\right)} .
\end{aligned}
$$

Appendix C. Proof of Proposition 3.7. We start by proving that the operator family $(\mathcal{A}(s))^{-1}: \widetilde{H}^{-1}\left(\Omega_{c}\right) \rightarrow H^{1}\left(\Omega_{c}\right), s \in \mathbb{C}^{+}$, where

$$
\langle\mathcal{A}(s) q, v\rangle_{\Omega_{c}}=a(q, v), \text { see }(3.4)
$$

is holomorphic in $\mathbb{C}^{+}$.

Step 1. Analyticity of $(\mathcal{A}(s))^{-1}$ 
1. First, let us show that for all $q, v \in H^{1}\left(I_{c}\right)$, the function $s \mapsto a_{m}(q, v)$, cf. (3.9), is analytic in $\mathbb{C}^{+}$. For this we consider different terms in the explicit expression for $a_{m}(q, v)$, cf. (3.9). The holomorphicity is not immediate for the third term in (3.9) (because the coefficients in its expansion into Laurent series will depend on $\sigma)$. However, by Lebesgue's dominated convergence theorem,

$$
\frac{d}{d s} \int_{I_{c}}\left(1+\frac{\sigma}{s}\right)^{-1} \partial_{x} q \overline{\partial_{x} v}=\int_{I_{c}} \frac{d}{d s}\left(1+\frac{\sigma}{s}\right)^{-1} \partial_{x} q \overline{\partial_{x} v}=\int_{I_{c}} \frac{\sigma}{(s+\sigma)^{2}} \partial_{x} q \overline{\partial_{x} v},
$$

i.e. $\int_{I_{c}}\left(1+\frac{\sigma}{s}\right)^{-1} \partial_{x} q \overline{\partial_{x} v}$ is $\mathbb{C}$-derivable in $\mathbb{C}^{+}$.

We conclude that $s \mapsto a_{m}(q, v)$ is holomorphic in $\mathbb{C}^{+}$for all $q, v \in H^{1}\left(\Omega_{c}\right)$.

2. Let us show that for all $q, v \in H^{1}\left(\Omega_{c}\right)$, the following series defines an analytic in $\mathbb{C}^{+}$function:

$$
\langle\mathcal{A}(s) q, v\rangle_{\Omega_{c}}=a(q, v)=\sum_{m=0}^{\infty} a_{m}\left(q_{m}, v_{m}\right)
$$

Because weak holomorphicity is equivalent to the (norm-)holomorphicity, see [48, Theorem III.3.12], this will yield that the family $\mathcal{A}: \mathbb{C}^{+} \rightarrow \mathcal{L}\left(H^{1}\left(\Omega_{c}\right), \tilde{H}^{-1}\left(\Omega_{c}\right)\right)$ is holomorphic. Let us demonstrate that (C.1) is holomorphic. Because each $a_{m}(q, v): \mathbb{C}^{+} \rightarrow \mathbb{C}$ is holomorphic, it suffices to show that the series in (C.1) converges uniformly on all compact subsets $K$ of $\mathbb{C}^{+}$. By (B.11),

$$
\begin{aligned}
\sup _{s \in K}\left|a_{m}\left(q_{m}, v_{m}\right)\right| & \leq C_{K}\left(\left(1+\lambda_{m}^{2}\right)\left\|q_{m}\right\|_{L^{2}\left(I_{c}\right)}\left\|v_{m}\right\|_{L^{2}\left(I_{c}\right)}\right. \\
& \left.+\left\|\partial_{x} q_{m}\right\|_{L^{2}\left(I_{c}\right)}\left\|\partial_{x} v_{m}\right\|_{L^{2}\left(I_{c}\right)}\right),
\end{aligned}
$$

and hence by the Cauchy-Schwarz inequality,

$$
\begin{aligned}
\sup _{s \in K} \sum_{m=N}^{\infty}\left|a_{m}\left(q_{m}, v_{m}\right)\right| & \leq C_{K}\left(\sum_{m=N}^{\infty}\left(\left(1+\lambda_{m}^{2}\right)\left\|q_{m}\right\|_{L^{2}\left(I_{c}\right)}^{2}+\|\left.\partial_{x} q_{m}\right|_{L^{2}\left(I_{c}\right)} ^{2}\right)\right)^{\frac{1}{2}} \\
& \times\left(\sum_{m=N}^{\infty}\left(\left(1+\lambda_{m}^{2}\right)\left\|v_{m}\right\|_{L^{2}\left(I_{c}\right)}^{2}+\left\|\partial_{x} v_{m}\right\|_{L^{2}\left(I_{c}\right)}^{2}\right)\right)^{\frac{1}{2}} .
\end{aligned}
$$

We then conclude about the uniform convergence of the series (C.1).

3. Because $(\mathcal{A}(s))^{-1} \in \mathcal{L}\left(H^{1}\left(\Omega_{c}\right), \widetilde{H}^{-1}\left(\Omega_{c}\right)\right)$ is well-defined for all $s \in \mathbb{C}^{+}$, see Proposition 3.5, by holomorphicity of $\mathcal{A}(s), \mathcal{A}: \mathbb{C}^{+} \rightarrow \mathcal{L}\left(H^{1}\left(\Omega_{c}\right), \widetilde{H}^{-1}\left(\Omega_{c}\right)\right)$ is a holomorphic operator-valued function in $\mathbb{C}^{+}$, see [48, Section VIII.1.1, p. 365].

Step 2. Analyticity of $\hat{u}^{\sigma}: \mathbb{C}^{+} \rightarrow H^{1}\left(\Omega_{c}\right)$. Remark that $\widetilde{F}:=\hat{f}_{s}: \mathbb{C}^{+} \rightarrow \widetilde{H}^{-1}$ is holomorphic in $\mathbb{C}^{+}$, by Theorem 3.4. Then $\hat{u}^{\sigma}(s)=\mathcal{A}^{-1}(s) \widetilde{F}(s)$ is holomorphic in $\mathbb{C}^{+}$as $H^{1}\left(\Omega_{c}\right)$-valued function.

\section{Appendix D. Proof of Theorem 3.9.}

Proof. Existence. By construction of the DtN maps, $\left.\hat{u}^{\sigma}\right|_{\Omega}$, where $\hat{u}^{\sigma}$ solves the well-posed (Proposition 3.5) problem (3.3), satisfies (3.26). The uniqueness follows by the same energy argument as the one used in the proof of Lemma 3.6 (see also 
[47], where a similar argument is used). Taking $\hat{f}_{s} \equiv 0$ and decomposing the solution of (3.26) into the modes yields

$$
\begin{aligned}
& \left(s^{2}+\lambda_{m}^{2}\right) \hat{u}_{\Omega, m}^{\sigma}-\partial_{x}^{2} \hat{u}_{\Omega, m}^{\sigma}=0 \quad \text { in } I, \\
& \partial_{x} \hat{u}_{\Omega, m}^{\sigma}( \pm a)=\mp \boldsymbol{T}_{\sigma, m}^{ \pm}(s) \hat{u}_{\Omega, m}^{\sigma}( \pm a) .
\end{aligned}
$$

Testing the above with $\hat{u}_{\Omega, m}^{\sigma}$ results in:

$$
\begin{aligned}
\left(s^{2}+\lambda_{m}^{2}\right) \int_{I}\left|\hat{u}_{\Omega, m}^{\sigma}\right|^{2} & +\int_{I}\left|\partial_{x} \hat{u}_{\Omega, m}^{\sigma}\right|^{2} \\
& +\boldsymbol{T}_{\sigma, m}^{+}\left|\hat{u}_{\Omega, m}^{\sigma}(a)\right|^{2}+\boldsymbol{T}_{\sigma, m}^{-}\left|\hat{u}_{\Omega, m}^{\sigma}(-a)\right|^{2}=0 .
\end{aligned}
$$

By construction of $\boldsymbol{T}_{\sigma}^{+}(s)$, cf. (3.16), from integration by parts applied to (3.17), we obtain, for any $\bar{v}_{m} \in H^{1}((a, a+L))$ :

$$
\begin{aligned}
\boldsymbol{T}_{\sigma, m}^{+}(s) G_{m}(a) \bar{v}_{m}(a) & =\left(s^{2}+\lambda_{m}^{2}\right) \int_{a}^{a+L}\left(1+\frac{\sigma}{s}\right) G_{m}(x) \bar{v}_{m}(x) \\
& +\int_{a}^{a+L}\left(1+\frac{\sigma}{s}\right)^{-1} \partial_{x} G_{m}(x) \partial_{x} \bar{v}_{m}(x) .
\end{aligned}
$$

A similar expression holds for $\boldsymbol{T}_{\sigma, m}^{-}$. Taking $G_{m}(a)=g_{m}=1$ in (3.16), and using the argument of the proof of Lemma 3.6, we obtain:

- $\operatorname{Re}\left(\boldsymbol{T}_{\sigma, m}^{ \pm}(s)\left|G_{m}( \pm a)\right|^{2}\right)=\operatorname{Re}\left(\boldsymbol{T}_{\sigma, m}^{ \pm}(s)\right) \geq 0$, when $\lambda_{m}^{2}>s_{i}^{2}$

- $\operatorname{Re}\left(\bar{s}_{\sigma, m}^{ \pm}(s)\right) \geq 0$, when $\lambda_{m}^{2} \leq s_{i}^{2}$.

It is then easy to see that

- when $\lambda_{m}^{2}>s_{i}^{2}$, taking the real part of (D.1) results in

$$
\left(s_{r}^{2}-s_{i}^{2}+\lambda_{m}^{2}\right) \int_{I}\left|\hat{u}_{\Omega, m}^{\sigma}\right|^{2}+\int_{I}\left|\partial_{x} \hat{u}_{\Omega, m}^{\sigma}\right|^{2} \leq 0, \quad \text { and thus } \hat{u}_{\Omega, m}^{\sigma}=0 .
$$

- when $\lambda_{m}^{2}<s_{i}^{2}$, multiplying (D.1) with $\bar{s}$ and taking the real part allows to conclude that $\hat{u}_{\Omega, m}^{\sigma}=0$.

We have thus proven the uniqueness of the solution.

\section{REFERENCES}

[1] S. Abarbanel and D. Gottlieb, A mathematical analysis of the PML method, J. Comput. Phys., 134 (1997), pp. 357-363.

[2] S. Abarbanel, D. Gottlieb, and J. S. Hesthaven, Well-posed perfectly matched layers for advective acoustics, J. Comput. Phys., 154 (1999), pp. 266-283.

[3] T. Abboud, P. Joly, J. Rodríguez, and I. Terrasse, Coupling discontinuous Galerkin methods and retarded potentials for transient wave propagation on unbounded domains, J. Comput. Phys., 230 (2011), pp. 5877-5907.

[4] D. Appelö, T. Hagstrom, and G. Kreiss, Perfectly matched layers for hyperbolic systems: general formulation, well-posedness, and stability, SIAM J. Appl. Math., 67 (2006), pp. 123.

[5] S. Asvadurov, V. Druskin, M. N. Guddati, and L. Knizhnerman, On optimal finitedifference approximation of PML, SIAM J. Numer. Anal., 41 (2003), pp. 287-305.

[6] D. Baffet, T. Hagstrom, And D. Givol, Double absorbing boundary formulations for acoustics and elastodynamics, SIAM J. Sci. Comput., 36 (2014), pp. A1277-A1312. 
[7] D. H. Baffet, M. J. Grote, S. Imperiale, and M. Kachanovska, Energy decay and stability of a perfectly matched layer for the wave equation, J. Sci. Comput., 81 (2019), pp. 22372270.

[8] A. Bamberger, P. Joly, And J. E. Roberts, Second-order absorbing boundary conditions for the wave equation: a solution for the corner problem, SIAM J. Numer. Anal., 27 (1990), pp. 323-352.

[9] L. Banjai, C. Lubich, And F.-J. SAyas, Stable numerical coupling of exterior and interior problems for the wave equation, Numer. Math., 129 (2015), pp. 611-646.

[10] E. Bécache, A.-S. Bonnet-Ben Dhia, and G. Legendre, Perfectly matched layers for the convected Helmholtz equation, SIAM J. Numer. Anal., 42 (2004), pp. 409-433.

[11] E. Bécache, A.-S. Bonnet-Ben Dhia, And G. Legendre, Perfectly matched layers for timeharmonic acoustics in the presence of a uniform flow, SIAM J. Numer. Anal., 44 (2006), pp. 1191-1217.

[12] E. BÉcache, S. FAuqueux, And P. Joly, Stability of perfectly matched layers, group velocities and anisotropic waves, J. Comput. Phys., 188 (2003), pp. 399-433.

[13] E. BÉCAChe AND P. Joly, On the analysis of Bérenger's perfectly matched layers for Maxwell's equations, M2AN Math. Model. Numer. Anal., 36 (2002), pp. 87-119.

[14] E. BÉCAChe, P. Joly, And M. Kachanovska, Stable perfectly matched layers for a cold plasma in a strong background magnetic field, J. Comput. Phys., 341 (2017), pp. 76-101.

[15] E. BÉCACHe, P. Joly, AND V. Vinoles, On the analysis of perfectly matched layers for a class of dispersive media and application to negative index metamaterials, Math. Comp., 87 (2018), pp. $2775-2810$.

[16] E. BÉCAChe AND M. KaChanovska, Stable perfectly matched layers for a class of anisotropic dispersive models. Part I: Necessary and sufficient conditions of stability, ESAIM Math. Model. Numer. Anal., 51 (2017), pp. 2399-2434.

[17] J.-P. Berenger, A perfectly matched layer for the absorption of electromagnetic waves, J. Comput. Phys., 114 (1994), pp. 185-200.

[18] J.-P. BERENGER, Three-dimensional perfectly matched layer for the absorption of electromagnetic waves, J. Comput. Phys., 127 (1996), pp. 363-379.

[19] A.-S. Bonnet-Ben Dhia, S. Fliss, and A. Tonnoir, The halfspace matching method: a new method to solve scattering problems in infinite media, J. Comput. Appl. Math., 338 (2018), pp. $44-68$.

[20] J. H. Bramble and J. E. Pasciak, Analysis of a finite element PML approximation for the three dimensional time-harmonic Maxwell problem, Math. Comp., 77 (2008), pp. 1-10.

[21] J. H. Bramble and J. E. Pasciak, Analysis of Cartesian PML approximation to the three dimensional electromagnetic wave scattering problem, Int. J. Numer. Anal. Model., 9 (2012), pp. 543-561.

[22] J. H. Bramble and J. E. Pasciak, Analysis of a Cartesian PML approximation to acoustic scattering problems in $\mathbb{R}^{2}$ and $\mathbb{R}^{3}$, J. Comput. Appl. Math., 247 (2013), pp. 209-230.

[23] J. H. Bramble, J. E. Pasciak, and D. Trenev, Analysis of a finite PML approximation to the three dimensional elastic wave scattering problem, Math. Comp., 79 (2010), pp. 2079-2101.

[24] Z. Chen And W. Zheng, PML method for electromagnetic scattering problem in a two-layer medium, SIAM J. Numer. Anal., 55 (2017), pp. 2050-2084.

[25] A. Chern, A reflectionless discrete perfectly matched layer, J. Comput. Phys., 381 (2019), pp. 91-109.

[26] F. Collino, High order absorbing boundary conditions for wave propagation models: straight line boundary and corner cases, in Second International Conference on Mathematical and Numerical Aspects of Wave Propagation (Newark, DE, 1993), SIAM, Philadelphia, PA, 1993, pp. 161-171.

[27] F. Collino And P. Monk, The perfectly matched layer in curvilinear coordinates, SIAM J. Sci. Comput., 19 (1998), pp. 2061-2090.

[28] F. Collino And P. B. Monk, Optimizing the perfectly matched layer, vol. 164, 1998, pp. 157171. Exterior problems of wave propagation (Boulder, CO, 1997; San Francisco, CA, 1997).

[29] R. Dautray and J.-L. Lions, Mathematical analysis and numerical methods for science and technology. Vol. 3, Springer-Verlag, Berlin, 1990. Spectral theory and applications, With the collaboration of Michel Artola and Michel Cessenat, Translated from the French by John C. Amson.

[30] R. Dautray and J.-L. Lions, Mathematical analysis and numerical methods for science and technology. Vol. 5, Springer-Verlag, Berlin, 1992. Evolution problems. I, With the collaboration of Michel Artola, Michel Cessenat and Hélène Lanchon, Translated from the French by Alan Craig.

[31] E. Demaldent and S. Imperiale, Perfectly matched transmission problem with absorbing lay- 
ers: application to anisotropic acoustics in convex polygonal domains, Internat. J. Numer. Methods Engrg., 96 (2013), pp. 689-711.

[32] J. Diaz And P. Joly, A time domain analysis of PML models in acoustics, Comput. Methods Appl. Mech. Engrg., 195 (2006), pp. 3820-3853.

[33] K. Duru And G. Kreiss, On the accuracy and stability of the perfectly matched layer in transient waveguides, J. Sci. Comput., 53 (2012), pp. 642-671.

[34] B. Engquist And A. MAJda, Absorbing boundary conditions for the numerical simulation of waves, Math. Comp., 31 (1977), pp. 629-651.

[35] S. D. GEDNEY, An anisotropic perfectly matched layer-absorbing medium for the truncation of fdtd lattices, IEEE transactions on Antennas and Propagation, 44 (1996), pp. 1630-1639.

[36] M. J. Grote And I. Sim, Efficient pml for the wave equation, 2010, https://arxiv.org/abs/ 1001.0319.

[37] T. Hagstrom, D. Givoli, D. Rabinovich, and J. Bielak, The double absorbing boundary method, J. Comput. Phys., 259 (2014), pp. 220-241.

[38] T. Hagstrom, A. Mar-Or, And D. Givoli, High-order local absorbing conditions for the wave equation: extensions and improvements, J. Comput. Phys., 227 (2008), pp. 3322-3357.

[39] T. Hagstrom and T. Warburton, A new auxiliary variable formulation of high-order local radiation boundary conditions: corner compatibility conditions and extensions to first-order systems, vol. 39, 2004, pp. 327-338. New computational methods for wave propagation.

[40] T. Hagstrom, T. Warburton, and D. Givoli, Radiation boundary conditions for timedependent waves based on complete plane wave expansions, J. Comput. Appl. Math., 234 (2010), pp. 1988-1995.

[41] L. Halpern, S. Petit-Bergez, and J. Rauch, The analysis of matched layers, Confluentes Math., 3 (2011), pp. 159-236.

[42] L. Halpern And J. RAUCh, Error analysis for absorbing boundary conditions, Numer. Math., 51 (1987), pp. 459-467.

[43] R. L. Higdon, Numerical absorbing boundary conditions for the wave equation, Math. Comp., 49 (1987), pp. 65-90.

[44] T. Hohage and L. Nannen, Hardy space infinite elements for scattering and resonance problems, SIAM J. Numer. Anal., 47 (2009), pp. 972-996.

[45] F. Q. Hu, A stable, perfectly matched layer for linearized Euler equations in unsplit physical variables, J. Comput. Phys., 173 (2001), pp. 455-480.

[46] Y. Huang, H. JiA, AND J. LI, Analysis and application of an equivalent Berenger's PML model, J. Comput. Appl. Math., 333 (2018), pp. 157-169.

[47] P. Joly and M. Kachanovska, Transparent boundary conditions for wave propagation in fractal trees: Convolution quadrature approach. Submitted.

[48] T. Kato, Perturbation theory for linear operators, Springer-Verlag, Berlin-New York, second ed., 1976. Grundlehren der Mathematischen Wissenschaften, Band 132.

[49] D. S. Katz, E. T. Thiele, AND A. TAFlove, Validation and extension to three dimensions of the berenger pml absorbing boundary condition for $f d$-td meshes, IEEE microwave and guided wave letters, 4 (1994), pp. 268-270.

[50] S. KIM, Error analysis of PML-FEM approximations for the Helmholtz equation in waveguides, ESAIM Math. Model. Numer. Anal., 53 (2019), pp. 1191-1222.

[51] S. KIM AND J. E. PASCIAK, Analysis of a Cartesian PML approximation to acoustic scattering problems in $\mathbb{R}^{2}$, J. Math. Anal. Appl., 370 (2010), pp. 168-186.

[52] A. Modave, E. Delhez, and C. Geuzaine, Optimizing perfectly matched layers in discrete contexts, Internat. J. Numer. Methods Engrg., 99 (2014), pp. 410-437.

[53] A. Modave, C. Geuzaine, and X. Antoine, Corner treatments for high-order local absorbing boundary conditions in high-frequency acoustic scattering, J. Comput. Phys., 401 (2020), pp. 109029, 24.

[54] F. NATAF, A new approach to perfectly matched layers for the linearized Euler system, J. Comput. Phys., 214 (2006), pp. 757-772.

[55] A. PAZY, Semigroups of linear operators and applications to partial differential equations, vol. 44 of Applied Mathematical Sciences, Springer-Verlag, New York, 1983.

[56] F.-J. SAYAS, Retarded potentials and time domain boundary integral equations, vol. 50 of Springer Series in Computational Mathematics, Springer, [Cham], 2016. A road map.

[57] F. L. Teixeira And W. C. Chew, General closed-form pml constitutive tensors to match arbitrary bianisotropic and dispersive linear media, IEEE Microwave and Guided Wave Letters, 8 (1998), pp. 223-225.

[58] F. Trèves, Basic linear partial differential equations, Academic Press, New York-London, 1975. Pure and Applied Mathematics, Vol. 62. 